\title{
Tracking multiple maneuvering targets hidden in the DBZ based on the MM-PHD filter
}

This paper was downloaded from TechRxiv (https://www.techrxiv.org).

\section{LICENSE}

CC BY 4.0

\section{SUBMISSION DATE / POSTED DATE}

08-07-2020 / 09-07-2020

\section{CITATION}

Wu, Weihua (2020): Tracking multiple maneuvering targets hidden in the DBZ based on the MM-PHD filter. TechRxiv. Preprint. https://doi.org/10.36227/techrxiv.12624599.v1

DOI 


\title{
Tracking multiple maneuvering targets hidden in the DBZ based on the MM-PHD filter
}

\author{
Weihua Wu, Weijian Liu, Hemin Sun, Yichao Cai, Jiajun Xiong
}

\begin{abstract}
For a ground moving target indication (GMTI) radar, the presence of Doppler blind zone (DBZ) results in many short tracks with frequent label switching, which seriously deteriorates the tracking performance. When the DBZ masking is coupled with targets maneuvering, tracking multiple maneuvering targets hidden in the DBZ becomes very challenging, which is reflected in the fact that there is no public research on this issue. To overcome this complicated problem, we propose a practical and fully functional GMTI multi-maneuvering-target tracker based on the multiple model probability hypothesis density (MM-PHD) filter. Unlike the standard MM-PHD filter, the proposed tracker utilizes the Doppler information and incorporates the minimum detectable velocity (MDV) to suppress the DBZ masking. Furthermore, to cope with the problems of the fixed initiation and no label output of the standard MM-PHD filter, the resulting MMPHD filter with the Doppler and MDV information is augmented with measurement-driven adaptive track initiation and track label propagation, which are necessary for a practical tracker and also required for evaluating the overall GMTI tracking performance. Finally, numerical examples show that the proposed tracker outperforms significantly the existing ones, thus verifying its effectiveness.
\end{abstract}

Index Terms - Ground moving target indication (GMTI), Multi-maneuvering-target tracking, Doppler blind zone (DBZ), Multiple model probability hypothesis density (MM-PHD), Minimum detectable velocity (MDV).

\section{NOMENCLATURE}

List of Acronyms

$\begin{array}{ll}\text { ATI } & \text { Adaptive track initiation } \\ \text { CPHD } & \text { Cardinalized PHD } \\ \text { DBZ } & \text { Doppler blind zone } \\ \text { GLMB } & \text { Generalized LMB } \\ \text { GM } & \text { Gaussian mixture } \\ \text { GMTI } & \text { Ground moving target indication } \\ \text { LMB } & \text { Labeled MB } \\ \text { MB } & \text { Multi-Bernoulli } \\ \text { MDV } & \text { Minimum detectable velocity } \\ \text { MM } & \text { Multiple model } \\ \text { MMTT } & \text { Multi-maneuvering-target tracking } \\ \text { MTT } & \text { Multi-target tracking }\end{array}$

Manuscript received January 07, 2020; revised XX XX, 2020; accepted XX XX, 2020. Date of publication XX XX, 2020; date of current version XX XX, 2020. The associate editor coordinating the review of this manuscript and approving it for publication was Prof. XX. This work was supported in part by the National Natural Science Foundation of China under Grant 61601510, in
OSPA Optimal sub-pattern assignment

OSPA-T OSPA for tracks

PHD Probability hypothesis density

RFS Random finite set

TLP Track label propagation

\section{INTRODUCTION}

GOR a ground moving target indication (GMTI) radar, the existence of Doppler blind zone (DBZ) is a main challenge

[1]. The DBZ arises from the clutter cancellation to separate moving target returns from the clutter background. Hence, lowDoppler targets, whose Doppler magnitudes fall below the minimum detectable velocity (MDV), can easily be masked by such a blind zone. To clarify the DBZ masking problem, we first analyze it in detail.

\section{A. DBZ masking problem for GMTI tracking}

For a GMTI radar, the detection probability $p_{D}(\boldsymbol{\xi})$, which is a function of target kinematic state $\boldsymbol{\xi}$, is strongly influenced by the well-known clutter notch $n_{c}$ (refer to [2] [3] for details), defined by

$$
n_{c}=n_{c}\left(\boldsymbol{\xi}, \boldsymbol{\xi}_{s}\right) \triangleq \frac{\dot{x}\left(x-x_{s}\right)+\dot{y}\left(y-y_{s}\right)+\dot{z}\left(z-z_{s}\right)}{\sqrt{\left(x-x_{s}\right)^{2}+\left(y-y_{s}\right)^{2}+\left(z-z_{s}\right)^{2}}}
$$

where $\boldsymbol{\xi}=\left[\begin{array}{llllll}x & y & z & \dot{x} & \dot{y} & \dot{z}\end{array}\right]^{\mathrm{T}}$ is the current kinematic state including the position part $(x, y, z)$ and the velocity part $(\dot{x}, \dot{y}, \dot{z})$. Similarly, $\boldsymbol{\xi}_{s}=\left[\begin{array}{llllll}x_{s} & y_{s} & z_{s} & \dot{x}_{s} & \dot{y}_{s} & \dot{z}_{s}\end{array}\right]^{\mathrm{T}}$ denotes the known sensor state at the same time.

The clutter notch not only suppresses clutters but also influences the low-Doppler target detection. Specifically, $p_{D}(\boldsymbol{\xi})$ approaches saturated value $p_{D}$ when $\left|n_{c}\right| \gg \mathrm{MDV}$, where $p_{D}$ is the conventional detection probability accounting for the directivity pattern and propagation effects of an antenna. However, $p_{D}(\boldsymbol{\xi})$ drops to zero when $\left|n_{c}\right|<\mathrm{MDV}$. This results in a series of missed detections and leads to many short trajectories whose track labels switch frequently, which ultimately deteriorates the tracking performance, as shown in Fig.1, where a GMTI sensor tracks two non-maneuvering targets under the clutter environment. Note that for each target's estimated trajectory, it sometimes misses estimations, and its label is switched since different colors represent different labels.

part by the Young Talent Support Project of China Association for Science and Technology under Grant 18-JCJQ-QT-008

The authors are with Air Force Early Warning Academy, Wuhan 430019, China. (e-mail: weihuawu1987@163.com). 


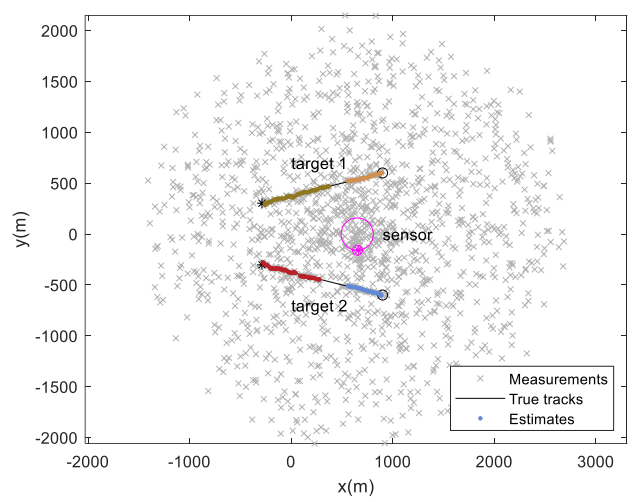

Fig.1. A typical tracking result for GMTI tracking.

However, the existing related works, which will be described in detail later, rarely paid attention to the label switching problem caused by the DBZ, since track labels are generally not considered. If track labels are not involved, a typical tracking result is depicted in Fig.2. The result can be obtained by using a general multi-target filter with the function of adaptive track initiation (ATI). Besides, a similar result can also be obtained by using an MDV incorporated multi-target filter (e.g., the filter in [4]) specially designed for the DBZ masking, since the MDV information can be conducive to the preservation of targets' information when targets are inside the DBZ. Hence, once targets are outside the DBZ, the sensor can obtain measurements originated from these targets, and get their estimations again even the used filter is limited to fixed track initiation. In other words, if there is no label information, we cannot distinguish whether the re-initiation capability benefits from the ATI or benefits from the introduction of the MDV. Nevertheless, the difference between the two can be clearly indicated by incorporating label information. If the label information is incorporated, the result using the ATI will become Fig.1, while the result using the MDV will become Fig.3, which is exactly what we expect. Therefore, to assess the overall GMTI tracking performance, especially the track reinitiation or label switching performance, it is necessary to account for the use of both the label and MDV information.

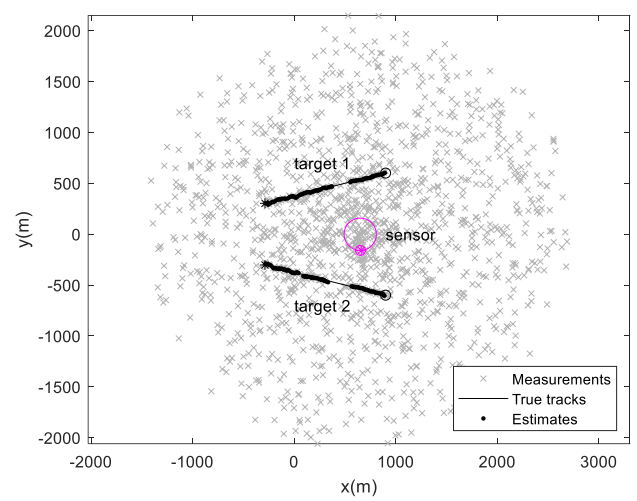

Fig.2. A typical result without considering track labels.

Of course, the ATI functionality is also necessary for a practical tracker. Besides, with comparison to the above nonmaneuvering multi-target tracking scenario, one may be more interested in tracking multiple maneuvering targets hidden in

\footnotetext{
${ }^{1}$ The multi-Bernoulli (MB) filter is the abbreviation for the cardinality balanced multi-target multi-Bernoulli (CBMeMBer) filter.
}

the DBZ. This is a very challenging topic and is still a research gap. Hence, it is the motivation of this paper.

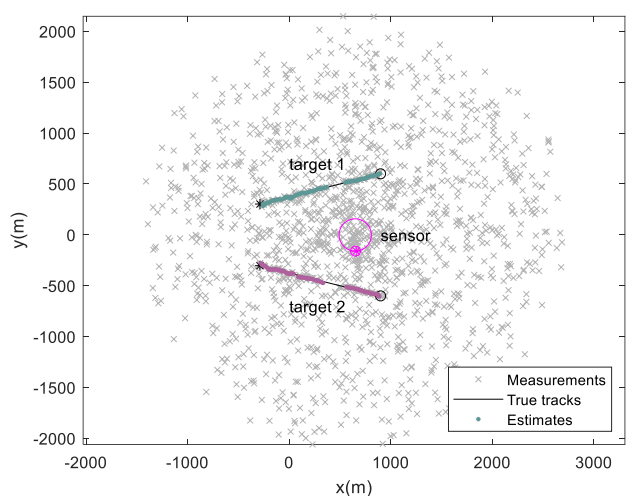

Fig.3. A typical result considering the label and MDV exploitation (and ATI).

In summary, for GMTI tracking, we need to comprehensively consider at least a multi-maneuvering-target filter, the use of MDV, ATI, and track label propagation (TLP). Finally, when considering these problems comprehensively, how to evaluate the overall GMTI tracking performance, especially the label switching performance, is a problem to be solved. In our earlier study [4], in order to consider the track reinitiation performance, we deliberately set the filter as a fixed track initiation, and then use the circular position error probability (CPEP) metric [5] to evaluate the performance. However, when the filter has the ATI function, it is obviously not appropriate to use this metric. Unfortunately, there is no open research on evaluating label switching caused by the DBZ, so it is necessary to find a suitable performance metric.

\section{B. Related works}

This paper focuses on the DBZ masking problem for GMTI tracking. There exist many approaches to dealing with the DBZ masking problem, such as [1], [2], [6-23], but the vast majority of those are oriented to tracking a single (non-maneuvering) target or multiple (non-maneuvering) targets in the presence of the DBZ. To avoid repetition, refer to our earlier studies [4] and the references listed therein for a detailed description.

In practice, the GMTI sensor inevitably faces maneuvering targets. Actually, the maneuvering-target tracking, especially the multi-maneuvering-target tracking (MMTT), has been a core difficult problem in the field of target tracking. The multiple models (MM) approach has proven to be a popular and effective tool for single-maneuvering-target tracking [24]. For the MMTT, the MM approach is usually combined with conventional multi-target tracking (MTT) techniques, such as multiple hypothesis tracking (MHT) [25], joint probabilistic data association (JPDA) [26], etc. However, these MTT algorithms are based on the traditional random vector framework. As promising candidates, the random finite set (RFS)-based approaches have attracted significant attention. Under the RFS framework, many well-known MTT algorithms have been developed, including the probability hypothesis density (PHD) [27], cardinalized PHD (CPHD) [28], multiBernoulli (MB) ${ }^{1}$ [29], labeled multi-Bernoulli (LMB) [30], marginalized GLMB (M-GLMB) ${ }^{2}$ [31], and the generalized

\footnotetext{
${ }^{2}$ The M-GLMB filter is the abbreviation for the marginalized $\delta$-GLMB (M $\delta$-GLMB) filter.
} 
labeled multi-Bernoulli (GLMB) ${ }^{3}$ filters $[32,33,34,35$ ] accompanied by two main implementations, i.e., the Gaussian mixture (GM) and sequential Monte Carlo (SMC) implementations. Meanwhile, many studies combine the MM with those RFS approaches to solve the MMTT problem, such as MM-PHD [36-39], MM-CPHD [40][41], MM-MB [42][43], MM-LMB [44], MM-GLMB [45-47], etc. However, these filters cannot be suitable for the DBZ masking case. For example, if the standard MM-PHD filter is directly applied to the GMTI tracking, once a target enters into the DBZ, the filter will miss the target, even though the target leaves outside the DBZ later, as shown by Fig.6(a) in the subsequent simulation. Additionally, even the standard MM-PHD filter is augmented with the ATI function, the estimated labels will be switched, as depicted in Fig.6(b). Hence, to cope with the DBZ masking, the RFS filters should be specially designed.

There are few studies on the DBZ masking with the RFS filters. In [3], the CPHD filter is applied to GMTI tracking, where the MDV information is incorporated into the detection probability model as done in [2] and the road map is integrated. Nevertheless, this work only utilized the MDV knowledge while ignored the Doppler information. Moreover, although the work also considered the history of a track, it is only used to plot a track. Hence, the work does not evaluate the label switching problem. In [48], the amplitude information is exploited based on the CPHD filter for GMTI tracking, but the DBZ masking problem is not involved there. [49] used the PHD filter to process the GMTI experimental data, but no detailed algorithm description is given there. Inspired by the detection probability model [2][3], we proposed a GM-PHD filter in [4] that exploited both the MDV and Doppler information. Nevertheless, this filter doesn't consider track labels and is deliberately set to be fixed track initiation to facilitate the evaluation of re-initiation performance as mentioned earlier.

Especially, the above works including ours don't consider target freely maneuvering. In fact, when the target maneuvering is coupled with the DBZ masking, the tracking task becomes very challenging. The challenge can be reflected from the fact that there exists only (non-maneuvering) multi-target tracking (as mentioned earlier) or single-maneuvering-target tracking, but there is no open research on tracking multiple maneuvering targets in Doppler blind zone. For instance, in [50, 51], a interacting multiple model (IMM) particle filter was proposed to track a single maneuvering target. In [52], a GM approach from [12] was extended to track a maneuvering target in clutter, based on the IMM [24] and the probabilistic data association (PDA) [53] techniques. Hence, these researches are limited to relatively simple single-maneuvering-target tracking. Therefore, tracking multiple maneuvering targets hidden in the DBZ is a brand-new but very practical problem with great research value.

This paper aims to this problem. Our purpose is to overcome the shortcomings of [4]. Specifically, the filter in [4] is extended to its multiple model version to adapt to the case of maneuvering targets. Furthermore, the obtained filter is enhanced by more practical measurement-driven ATI and track label propagation (TLP). The main contributions are as follows.

\footnotetext{
${ }^{3}$ The GLMB filter is also named Vo-Vo filter by Mahler.

${ }^{4}$ For clarity the time index $k$ will sometimes be omitted and the prediction is indicated by the subscripts "+" when there is no risk of confusion.
}

1) We clearly point out that it is needed to comprehensively consider the multi-maneuvering-target filter, MDV exploitation, ATI, and TLP, which are required for not only a practical GMTI tracker but also the evaluation of the overall GMTI tracking performance.

2) we extend our previous work [4] and propose a specially designed multi-maneuvering-target tracker based on the MMPHD filter [37] for the DBZ masking problem. Concretely, we derive a novel MM-PHD filter with the MDV and Doppler information by applying the realistic GMTI measurement model (including the MDV incorporated detection probability model) to the MM-PHD filter. Furthermore, we present the measurement-driven ATI and TLP approaches for the resulting filter.

3) we prove through simulation that the newly developed OSPA $^{(2)}$ [54] is not suitable for assessing the performance of label switching caused by the DBZ. Hence, we find another one, OSPA-T [55], used to evaluate the label switching performance.

In a word, we propose a practical and fully functional tracker for the GMTI MMTT. This work is an attempt to track multiple maneuvering targets hidden in the DBZ. The basic idea here has been presented at a conference in [56], and has been applied to the MM-GLMB filter [57].

The remainder of the paper is organized as follows. Section II describes the system model for the MMTT with a GMTI radar. Section III summarizes the standard MM-PHD filter, which is the foundation of the proposed tracker. Section IV presents the proposed specially designed tracker. Section V provides numerical studies to illustrate its effectiveness. Finally, conclusions are given in Sections VI.

\section{SYSTEM MODEL FOR THE MMTT WITH A GMTI RADAR}

Consider an MMTT scenario with a GMTI radar. Suppose at time $k-1$ there are $\left|X^{\prime}\right|$ targets, whose states are $X^{\prime}=\left\{\boldsymbol{x}_{1}^{\prime}, \boldsymbol{x}_{2}^{\prime}, \cdots, \boldsymbol{x}_{\left|X^{\prime}\right|}^{\prime}\right\}$ with $\boldsymbol{x}^{\prime}=\left[\boldsymbol{\xi}^{\prime}, \mu^{\prime}\right]$ for each $\boldsymbol{x}^{\prime} \in X^{\prime}{ }^{4}$, where $\xi^{\prime} \in \mathbb{X}$ and $\mu^{\prime} \in \mathbb{U}$ denote a (continuous) kinematic state and a (discrete) mode state, respectively, $\mathbb{X}$ and $\mathbb{U}$ denote the corresponding spaces, $\left|X^{\prime}\right|$ is the cardinality of a set $X^{\prime}$, the prime symbol $(\cdot)^{\prime}$ denotes a variable at the previous time (e.g., $k-1$ ). Hence, compared with the conventional MTT, a kinematic state is augmented with a mode variable for the MMTT. At the next time, new targets may appear, and some of previous targets may die with probability $1-p_{S}\left(\boldsymbol{x}^{\prime}\right)$, or continue to survive with survival probability $p_{S}\left(\boldsymbol{x}^{\prime}\right)^{5}$. If a target with state $x^{\prime}$ survives, it evolves to a new state $x$ with the following transition probability

$$
\phi\left(\boldsymbol{x} \mid \boldsymbol{x}^{\prime}\right)=\phi\left(\boldsymbol{\xi}, \mu \mid \boldsymbol{\xi}^{\prime}, \mu^{\prime}\right)=\phi\left(\boldsymbol{\xi} \mid \boldsymbol{\xi}^{\prime}, \mu\right) \tau\left(\mu \mid \mu^{\prime}\right)
$$

where $\phi\left(\boldsymbol{\xi} \mid \boldsymbol{\xi}^{\prime}, \mu\right)$ is the state transition probability density, and $\tau\left(\mu \mid \mu^{\prime}\right)$ denotes the mode transition probability to $\mu$ from $\mu^{\prime}$, which satisfies

$$
\sum_{\mu} \tau\left(\mu \mid \mu^{\prime}\right)=1
$$

\footnotetext{
${ }^{5}$ The target spawning case is omitted here.
} 
For multiple targets with state $X=\left\{\boldsymbol{x}_{1}, \boldsymbol{x}_{2}, \cdots, \boldsymbol{x}_{|X|}\right\}$ at time $k$, the measurement set received by a GMTI radar is represented as $Z=\left\{z_{1}, z_{2}, \cdots, z_{|Z|}\right\}$. Some of those measurements may come from Poisson clutters with intensity $\kappa$, and some may be originated from detected targets. It is assumed that each target with state $\boldsymbol{x}=[\boldsymbol{\xi}, \mu] \in X$ is detected with state-dependent detection probability $p_{D}(\boldsymbol{x})$. Here, it is reasonable to assume that the detection probability is independent on mode variable $\mu$, i.e., $p_{D}(\boldsymbol{x})=p_{D}(\boldsymbol{\xi})$. Conditioned on detection, the PDF of an observation $z \in Z$ originated from this target is denoted by likelihood function $g(\boldsymbol{z} \mid \boldsymbol{x})$. Generally, likelihood $g(\boldsymbol{z} \mid \boldsymbol{x})$ is usually derived by a measurement model. For the GMTI radar, the measurement model is given by [58][59]

$$
\begin{aligned}
\boldsymbol{z} & \triangleq \boldsymbol{z}_{p}=\left[\boldsymbol{y}_{p} ; y_{d}\right]=h\left(\boldsymbol{x}, \boldsymbol{\xi}_{s}\right)+\boldsymbol{n}_{p}=h\left(\boldsymbol{\xi}, \boldsymbol{\xi}_{s}\right)+\boldsymbol{n}_{p} \\
& =\left[\begin{array}{llll}
r_{m} & a_{m} & e_{m} & y_{d}
\end{array}\right]^{\mathrm{T}} \\
& =\left[\begin{array}{llll}
r & a & e & \dot{r}
\end{array}\right]^{\mathrm{T}}+\left[\begin{array}{lllll}
n_{r} & n_{a} & n_{e} & n_{d}
\end{array}\right]^{\mathrm{T}}
\end{aligned}
$$

where $\boldsymbol{\xi}$ and $\boldsymbol{\xi}_{s}$ are defined before, and

$$
[r, a, e]=\operatorname{Cart} 2 \operatorname{Polar}\left(\boldsymbol{\xi}, \boldsymbol{\xi}_{s}\right)
$$

$$
\begin{aligned}
\dot{r} & \triangleq h_{d}\left(\boldsymbol{\xi}, \boldsymbol{\xi}_{s}\right) \\
& =\left(\dot{x}-\dot{x}_{s}\right) \cos a \cos e+\left(\dot{y}-\dot{y}_{s}\right) \sin a \cos e+\left(\dot{z}-\dot{z}_{s}\right) \sin e \\
& =\frac{\left(x-x_{s}\right)\left(\dot{x}-\dot{x}_{s}\right)+\left(y-y_{s}\right)\left(\dot{y}-\dot{y}_{s}\right)+\left(z-z_{s}\right)\left(\dot{z}-\dot{z}_{s}\right)}{\sqrt{\left(x-x_{s}\right)^{2}+\left(y-y_{s}\right)^{2}+\left(z-z_{s}\right)^{2}}}
\end{aligned}
$$

In the above, a measurement $z$ is composed of a spherical coordinate $\boldsymbol{y}_{p}=\left[r_{m}, a_{m}, e_{m}\right]^{\mathrm{T}}$ and Doppler (or radial velocity) part $y_{d} ; r, a, e$ and $\dot{r}$ denote the true range, azimuth, elevation and Doppler of the target with respect to the sensor, respectively; measurement noise $\boldsymbol{n}_{p}$ follows a zero-mean Gaussian distribution with covariance matrix $\boldsymbol{R}_{p d}=\operatorname{blkdiag}\left(\boldsymbol{R}_{p}, \sigma_{d}^{2}\right)$, in which $\boldsymbol{R}_{p}=\operatorname{blkdiag}\left(\sigma_{r}^{2}, \sigma_{a}^{2}, \sigma_{e}^{2}\right)$ and the "blkdiag" represents a block diagonal matrix; Function Cart2Polar $(\cdot, \cdot)$ is used to transform Cartesian coordinates to polar coordinates, given in Appendix A. From (4), the concrete form of likelihood $g(z \mid x)$ can be calculated as

$$
g(\boldsymbol{z} \mid \boldsymbol{x})=g(\boldsymbol{z} \mid \boldsymbol{\xi}, \mu)=g(\boldsymbol{z} \mid \boldsymbol{\xi})=\mathcal{N}\left(\boldsymbol{z} ; h\left(\boldsymbol{\xi}, \boldsymbol{\xi}_{s}\right), \boldsymbol{R}_{p d}\right)
$$

where the measurement model is assumed to be independent on mode $\mu$, and $\mathcal{N}(\cdot ; \boldsymbol{m}, \boldsymbol{P})$ denotes a Gaussian density with mean $\boldsymbol{m}$ and covariance $\boldsymbol{P}$.

\section{MM-PHD FILTER}

In order to resolve the MMTT problem, we resort to the multiple model PHD (MM-PHD) filter. Based on some model assumptions (see [27] and [37] for details), by substituting the augmented state into the standard PHD filter [27] and extending the conventional inner product $\langle\alpha, \beta\rangle \triangleq \int \alpha(\boldsymbol{\xi}) \beta(\boldsymbol{\xi}) d \boldsymbol{\xi}$ (for realvalued functions $\alpha$ and $\beta$ ) or $\langle\alpha, \beta\rangle \triangleq \sum_{i=0}^{\infty} \alpha(i) \beta(i)$ (for real sequences $\alpha$ and $\beta$ ) to $\langle\alpha, \beta\rangle \triangleq \sum_{\mu} \int \alpha(\boldsymbol{\xi}, \mu) \beta(\boldsymbol{\xi}, \mu) d \boldsymbol{\xi}$ (for functions $\alpha$ and $\beta$ whose arguments consist of continuous and discrete variables), we can obtain the MM-PHD filter [37], which includes the following prediction and update equations:

$$
\begin{aligned}
v_{+}(\boldsymbol{\xi}, \mu)= & \sum_{\mu^{\prime}} \int p_{S}\left(\boldsymbol{\xi}^{\prime}, \mu^{\prime}\right) \phi\left(\boldsymbol{\xi}, \mu \mid \boldsymbol{\xi}^{\prime}, \mu^{\prime}\right) v\left(\boldsymbol{\xi}^{\prime}, \mu^{\prime}\right) d \boldsymbol{\xi}^{\prime}+v_{\gamma}(\boldsymbol{\xi}, \mu) \\
v(\boldsymbol{\xi}, \mu)= & {\left[1-p_{D}(\boldsymbol{\xi}, \mu)\right] v_{+}(\boldsymbol{\xi}, \mu)+} \\
& \sum_{z \in Z} \frac{p_{D}(\boldsymbol{\xi}, \mu) g(\boldsymbol{z} \mid \boldsymbol{\xi}, \mu) v_{+}(\boldsymbol{\xi}, \mu)}{\kappa(\boldsymbol{z})+\sum_{\tilde{\mu}} \int p_{D}(\tilde{\boldsymbol{\xi}}, \tilde{\mu}) g(\boldsymbol{z} \mid \tilde{\boldsymbol{\xi}}, \tilde{\mu}) v_{+}(\tilde{\boldsymbol{\xi}}, \tilde{\mu}) d \tilde{\boldsymbol{\xi}}}
\end{aligned}
$$

where $v$ denotes the prior intensity if its arguments are $\left(\boldsymbol{\xi}^{\prime}, \mu^{\prime}\right)$ or the posterior intensity if its arguments are $(\boldsymbol{\xi}, \mu), v_{+}$is the predicted intensity to time $k, v_{\gamma}$ represents the birth intensity at time $k$, which is generally given by a preset birth model [37]. The measurement set $Z$, survival probability $p_{S}$, transition density $\phi(\cdot \mid \cdot)$, detection probability $p_{D}(\cdot)$, and likelihood $g(\cdot \mid \cdot)$ are described before in Section II, $\kappa(\cdot)=\lambda_{C} p_{C}(\cdot)$ is the clutter intensity with clutter ratio (or expected number) $\lambda_{C}=\int \kappa(z) d z$ and clutter spatial distribution $p_{C}(\cdot)=\kappa(\cdot) / \lambda_{C}$ over the surveillance region.

Note that the standard MM-PHD filter is limited to fixed track initiation, and it cannot output track labels. Moreover, it doesn't account for the realistic GMTI measurement model, e.g., the polar and Doppler measurements, especially the DBZ masking. In the following, we extend the MM-PHD filter to a practical tracker to accommodate the GMTI DBZ masking problem.

\section{PROPOSED TRACKER}

Based on the above MM-PHD filter, the proposed tracker incorporates the (Doppler and) MDV information and has the ATI and TLP capabilities, in order to cope with such problems as the DBZ masking, fixed track initiation and no label output of the standard MM-PHD filter. Macroscopically, the proposed tracker consists of prediction, update, and ATI, all of which will be extended later to propagate track labels.

\section{A. Prediction}

Assume that each mode within mode space $\mathbb{U}$ follows a linear Gaussian model, i.e., the $\phi\left(\xi \mid \boldsymbol{\xi}^{\prime}, \mu\right)$ in (2) has the following form

$$
\phi\left(\boldsymbol{\xi} \mid \boldsymbol{\xi}^{\prime}, \mu\right)=\mathcal{N}\left(\boldsymbol{\xi} ; \boldsymbol{F}(\mu) \boldsymbol{\xi}^{\prime}, \boldsymbol{Q}(\mu)\right)
$$

where $\boldsymbol{F}(\mu)$ and $\boldsymbol{Q}(\mu)$ are the state transition matrix and process noise covariance conditioned on mode $\mu$, respectively.

Furthermore, if the prior intensity $v$ at time $k-1$ is a GM of the form

$$
\begin{aligned}
v\left(\boldsymbol{\xi}^{\prime}, \mu^{\prime}\right) & =\sum_{i=1}^{J^{\prime}\left(\mu^{\prime}\right)} w^{(i)}\left(\mu^{\prime}\right) \mathcal{N}\left(\boldsymbol{\xi}^{\prime} ; \boldsymbol{m}^{(i)}\left(\mu^{\prime}\right), \boldsymbol{P}^{(i)}\left(\mu^{\prime}\right)\right) \\
& =v_{S}\left(\boldsymbol{\xi}^{\prime}, \mu^{\prime}\right)+v_{\gamma}\left(\boldsymbol{\xi}^{\prime}, \mu^{\prime}\right)
\end{aligned}
$$

where $v_{S}\left(\boldsymbol{\xi}^{\prime}, \mu^{\prime}\right)$ is the prior survival intensity, obtained from measurement update (26) in Section IV.B, and $v_{\gamma}\left(\boldsymbol{\xi}^{\prime}, \mu^{\prime}\right)$ is the measurement-driven birth intensity, obtained from the ATI in Section IV.C, and they are denoted by

$$
\begin{gathered}
v_{S}\left(\boldsymbol{\xi}^{\prime}, \mu^{\prime}\right)=\sum_{i=1}^{J_{S}^{\prime}\left(\mu^{\prime}\right)} w_{S}^{(i)}\left(\mu^{\prime}\right) \mathcal{N}\left(\boldsymbol{\xi}^{\prime} ; \boldsymbol{m}_{S}^{(i)}\left(\mu^{\prime}\right), \boldsymbol{P}_{S}^{(i)}\left(\mu^{\prime}\right)\right) \\
v_{\gamma}\left(\boldsymbol{\xi}^{\prime}, \mu^{\prime}\right)=p_{\gamma}\left(\mu^{\prime}\right) \sum_{i=1}^{J_{\gamma}^{\prime}\left(\mu^{\prime}\right)} w_{\gamma}^{(i)}\left(\mu^{\prime}\right) \mathcal{N}\left(\boldsymbol{\xi}^{\prime} ; \boldsymbol{m}_{\gamma}^{(i)}\left(\mu^{\prime}\right), \boldsymbol{P}_{\gamma}^{(i)}\left(\mu^{\prime}\right)\right)
\end{gathered}
$$


respectively. Then the predicted intensity $v_{+}$to time $k$ is also a GM, given by

$$
\begin{aligned}
v_{+}(\boldsymbol{\xi}, \mu)= & v_{S,+}(\boldsymbol{\xi}, \mu)+v_{\gamma,+}(\boldsymbol{\xi}, \mu) \\
= & \sum_{i=1}^{J_{S}^{\prime}(\mu)} w_{S,+}^{(i)}(\mu) \mathcal{N}\left(\boldsymbol{\xi} ; \boldsymbol{m}_{S,+}^{(i)}(\mu), \boldsymbol{P}_{S,+}^{(i)}(\mu)\right) \\
& +\sum_{i=1}^{J_{\gamma}^{\prime}(\mu)} w_{\gamma,+}^{(i)}(\mu) \mathcal{N}\left(\boldsymbol{\xi} ; \boldsymbol{m}_{\gamma,+}^{(i)}(\mu), \boldsymbol{P}_{\gamma,+}^{(i)}(\mu)\right) \\
= & \sum_{i=1}^{J_{+}(\mu)} w_{+}^{(i)}(\mu) \mathcal{N}\left(\boldsymbol{\xi} ; \boldsymbol{m}_{+}^{(i)}(\mu), \boldsymbol{P}_{+}^{(i)}(\mu)\right)
\end{aligned}
$$

where

$$
\begin{aligned}
& v_{S,+}(\boldsymbol{\xi}, \mu)= \sum_{i=1}^{J_{S}^{\prime}(\mu)} w_{S,+}^{(i)}(\mu) \mathcal{N}\left(\boldsymbol{\xi} ; \boldsymbol{m}_{S,+}^{(i)}(\mu), \boldsymbol{P}_{S,+}^{(i)}(\mu)\right) \\
&= \sum_{\mu^{\prime}} \sum_{i=1}^{J_{S}^{\prime}\left(\mu^{\prime}\right)} w_{S,+}^{(i)}\left(\mu, \mu^{\prime}\right) \mathcal{N}\left(\boldsymbol{\xi} ; \boldsymbol{m}_{S,+}^{(i)}\left(\mu, \mu^{\prime}\right), \boldsymbol{P}_{S,+}^{(i)}\left(\mu, \mu^{\prime}\right)\right) \\
& {\left[\boldsymbol{m}_{S,+}^{(i)}\left(\mu, \mu^{\prime}\right), \boldsymbol{P}_{S,+}^{(i)}\left(\mu, \mu^{\prime}\right)\right]=\mathrm{KF}_{-} \mathrm{P}_{-} \mathrm{MM}\left(\boldsymbol{m}_{S}^{(i)}\left(\mu^{\prime}\right), \boldsymbol{P}_{S}^{(i)}\left(\mu^{\prime}\right), \boldsymbol{F}(\mu), \boldsymbol{Q}(\mu)\right) } \\
& w_{S,+}^{(i)}\left(\mu, \mu^{\prime}\right)=\tau\left(\mu \mid \mu^{\prime}\right) p_{S} w_{S}^{(i)}\left(\mu^{\prime}\right) \\
& v_{\gamma,+}(\boldsymbol{\xi}, \mu)=\sum_{i=1}^{J_{\gamma}^{\prime}(\mu)} w_{\gamma,+}^{(i)}(\mu) \mathcal{N}\left(\boldsymbol{\xi} ; \boldsymbol{m}_{\gamma,+}^{(i)}(\mu), \boldsymbol{P}_{\gamma,+}^{(i)}(\mu)\right) \\
&=\sum_{\mu^{\prime}} \sum_{i=1}^{J_{\gamma}^{\prime}\left(\mu^{\prime}\right)} w_{\gamma,+}^{(i)}\left(\mu, \mu^{\prime}\right) \mathcal{N}\left(\boldsymbol{\xi} ; \boldsymbol{m}_{\gamma,+}^{(i)}\left(\mu, \mu^{\prime}\right), \boldsymbol{P}_{\gamma,+}^{(i)}\left(\mu, \mu^{\prime}\right)\right) \\
& {\left[\boldsymbol{m}_{\gamma,+}^{(i)}\left(\mu, \mu^{\prime}\right), \boldsymbol{P}_{\gamma,+}^{(i)}\left(\mu, \mu^{\prime}\right)\right]=\mathrm{KF}_{-} \mathbf{P}_{-} \operatorname{MM}\left(\boldsymbol{m}_{\gamma}^{(i)}\left(\mu^{\prime}\right), \boldsymbol{P}_{\gamma}^{(i)}\left(\mu^{\prime}\right), \boldsymbol{F}(\mu), \boldsymbol{Q}(\mu)\right) } \\
& w_{\gamma,+}^{(i)}\left(\mu, \mu^{\prime}\right)=\tau\left(\mu \mid \mu^{\prime}\right) p_{S} w_{\gamma}^{(i)}\left(\mu^{\prime}\right) p_{\gamma}\left(\mu^{\prime}\right)
\end{aligned}
$$

In the above, survival probability $p_{S}\left(\boldsymbol{x}^{\prime}\right)$ is assumed to be independent of $\boldsymbol{x}^{\prime}$ for simplicity, and function "KF_P_MM", representing the MM version of the Kalman prediction operator, is summarized in Table I.

TABLE I

FUNCTION KF_P_MM

$\frac{\overline{\left[\boldsymbol{m}_{+}\left(\mu, \mu^{\prime}\right), \boldsymbol{P}_{+}\left(\mu, \mu^{\prime}\right)\right]=\text { KF_P_MM }\left(\boldsymbol{m}\left(\mu^{\prime}\right), \boldsymbol{P}\left(\mu^{\prime}\right), \boldsymbol{F}(\mu), \boldsymbol{Q}(\mu)\right)}}{m_{+}\left(\mu, \mu^{\prime}\right)=\boldsymbol{F}(\mu) \boldsymbol{m}\left(\mu^{\prime}\right)}$

$$
\boldsymbol{P}_{+}\left(\mu, \mu^{\prime}\right)=\boldsymbol{F}(\mu) \boldsymbol{P}\left(\mu^{\prime}\right) \boldsymbol{F}^{\mathrm{T}}(\mu)+\boldsymbol{Q}(\mu)
$$

$R \overline{e ~ m a \text { Note that the fixed birth intensity } v_{\gamma}(8) \text { is replaced }}$ by predicted birth intensity $v_{\gamma,+}(\cdot)(14)$, and initiation of associated parameters $w_{\gamma}^{(i)}\left(\mu^{\prime}\right), \boldsymbol{m}_{\gamma}^{(i)}\left(\mu^{\prime}\right), \boldsymbol{P}_{\gamma}^{(i)}\left(\mu^{\prime}\right), p_{\gamma}\left(\mu^{\prime}\right)$ and $J_{\gamma}^{\prime}\left(\mu^{\prime}\right)$ in (13) will be given in Section IV.C. Unlike the fixed track initiation in the standard MM-PHD filter, where the target birth process occurs in the prediction, it is suggested here that this process occurs after measurement update instead (see Section IV.C for details). We argue that the proposed scheme that track initiation is located after the update step has a better logic than the standard scheme that track initiation is located in the prediction.

\section{B. Update}

The prediction step is followed by measurement update once measurements are available. Before measurement update, we first carry out measurement transformation to Cartesian coordinates from polar coordinates.

\section{1) Measurement transformation}

For a polar measurement $\boldsymbol{y}_{p}=\left[r_{m}, a_{m}, e_{m}\right]^{\mathrm{T}}$ obtained from a GMTI radar at time $k$, given by (4), its corresponding Cartesian measurement $\boldsymbol{y}_{c}=\left[x_{m}, y_{m}, z_{m}\right]^{\mathrm{T}}$ can be computed via the nonlinear transformation $\boldsymbol{y}_{c}=\operatorname{Polar} 2 \operatorname{Cart}\left(\boldsymbol{y}_{p}, \boldsymbol{\xi}_{s}\right)$ in Appendix B, where $\boldsymbol{\xi}_{s}$ denotes the known sensor state. Meanwhile, given the covariance $\boldsymbol{R}_{p}$ of $\boldsymbol{y}_{p}$, the corresponding covariance $\boldsymbol{R}_{c}\left(\boldsymbol{y}_{p}\right)$ of $\boldsymbol{y}_{c}$ after measurement transformation is given by Cov_Polar2Cart in Appendix C.

Let $\boldsymbol{z} \leftarrow \boldsymbol{z}_{c}=\left[\boldsymbol{y}_{c} ; y_{d}\right]$, where $\boldsymbol{y}_{c}$ and $y_{d}$ denote the converted position measurement and the Doppler measurement, respectively. If $\boldsymbol{y}_{c}$ and $y_{d}$ are uncorrelated, likelihood $g(\boldsymbol{z} \mid \boldsymbol{x})$ (7) can be approximated as [60]

$$
g(\boldsymbol{z} \mid \boldsymbol{\xi}, \mu) \approx g\left(\boldsymbol{z}_{c} \mid \boldsymbol{\xi}, \mu\right)=g\left(\boldsymbol{y}_{c} \mid \boldsymbol{\xi}, \mu\right) g\left(y_{d} \mid \boldsymbol{\xi}, \mu\right)
$$
with

$$
\begin{aligned}
& g\left(\boldsymbol{y}_{c} \mid \boldsymbol{\xi}, \mu\right)=g\left(\boldsymbol{y}_{c} \mid \boldsymbol{\xi}\right)=\mathcal{N}\left(\boldsymbol{y}_{c} ; \boldsymbol{H}_{c} \boldsymbol{\xi}, \boldsymbol{R}_{c}\left(\boldsymbol{y}_{p}\right)\right) \\
& g\left(y_{d} \mid \boldsymbol{\xi}, \mu\right)=g\left(y_{d} \mid \boldsymbol{\xi}\right)=\mathcal{N}\left(y_{d} ; h_{d}(\boldsymbol{\xi}), \sigma_{d}^{2}\right)
\end{aligned}
$$

where $\boldsymbol{H}_{c}=\operatorname{diag}\left(\boldsymbol{I}_{3}, \boldsymbol{O}_{3}\right)$ is a position observation matrix, $\boldsymbol{I}_{n}$ and $\boldsymbol{O}_{n}$ denote $n \times n$ identity and zero matrices, respectively. Note that there exists a 1-1 relation between $\boldsymbol{y}_{c}$ and $\boldsymbol{y}_{p}$ in (22). Additionally, it is assumed that measurement model parameters are independent on mode $\mu$. When they depend on model $\mu$, we can simply substitute $\boldsymbol{H}_{c}=\boldsymbol{H}_{c}(\mu), \boldsymbol{R}_{c}\left(\boldsymbol{y}_{p}\right)=\boldsymbol{R}_{c}\left(\boldsymbol{y}_{p}, \mu\right)$, $h_{d}(\boldsymbol{\xi})=h_{d}(\boldsymbol{\xi}, \mu)$ into the above equations.

2) Measurement update

To suppress the DBZ masking problem, the measurement update utilizes the MDV information [61], which is an important parameter since the width of the DBZ is generally determined by the MDV. To this end, the MDV incorporated detection probability is adopted as follows [2][3][4]

$$
p_{D}(\boldsymbol{x})=p_{D}(\boldsymbol{\xi})=p_{D} \cdot\left[1-c_{f} \mathcal{N}\left(y_{f}\left(\hat{\boldsymbol{\xi}}_{+}\right) ; \boldsymbol{H}_{f}\left(\hat{\boldsymbol{\xi}}_{+}\right) \cdot \boldsymbol{\xi}, R_{f}\right)\right]
$$

with the fictitious measurement function

$$
y_{f} \triangleq y_{f}\left(\hat{\boldsymbol{\xi}}_{+}\right)=n_{c}\left(\hat{\boldsymbol{\xi}}_{+}\right)+\boldsymbol{H}_{f}\left(\hat{\boldsymbol{\xi}}_{+}\right) \hat{\boldsymbol{\xi}}_{+}
$$

and the corresponding fictitious measurement matrix $\boldsymbol{H}_{f}\left(\hat{\boldsymbol{\xi}}_{+}\right)$ given in Appendix D. In the above, $\hat{\boldsymbol{\xi}}_{+}$is the predicted state, $c_{f}=\mathrm{MDV} \sqrt{\pi / \log 2}$ is a normalization factor and $R_{f}=\mathrm{MDV}^{2} /(2 \log 2)$ is the variance of the fictitious measurement in the Doppler domain. Hence, the information on the clutter notch in (1) serves as fictitious measurements, and the MDV plays the role of the standard deviation for fictitious measurements [3].

Note that from (8) and (9) the detection probability only affects the update equation. Then, given the GM form of predicted intensity (14), by substituting the resulting likelihood function (21) and detection probability (24) into the MM-PHD update equation (9), applying successively the product formula for normal densities in Appendix E, and rearranging the terms, we obtain the GM update intensity as follows

$$
\begin{aligned}
& v(\boldsymbol{\xi}, \mu) \\
= & \sum_{j=1}^{J_{+}(\mu)} w_{0}^{(j)}(\mu) \mathcal{N}\left(\boldsymbol{\xi} ; \boldsymbol{m}_{0}^{(j)}(\mu), \boldsymbol{P}_{0}^{(j)}(\mu)\right)+ \\
& \sum_{j=1}^{J_{+}(\mu)} w_{f}^{(j)}(\mu) \mathcal{N}\left(\boldsymbol{\xi} ; \boldsymbol{m}_{f}^{(j)}(\mu), \boldsymbol{P}_{f}^{(j)}(\mu)\right)+ \\
& \sum_{z \in Z} \sum_{j=1}^{J_{+}(\mu)} \tilde{w}_{d}^{(j)}(\boldsymbol{z}, \mu) \mathcal{N}\left(\boldsymbol{\xi} ; \boldsymbol{m}_{d}^{(j)}(\boldsymbol{z}, \mu), \boldsymbol{P}_{d}^{(j)}(\boldsymbol{z}, \mu)\right)+ \\
& \sum_{z \in Z} \sum_{j=1}^{J_{+}(\mu)} \tilde{w}_{f}^{(j)}(\boldsymbol{z}, \mu) \mathcal{N}\left(\boldsymbol{\xi} ; \boldsymbol{m}_{f}^{(j)}(\boldsymbol{z}, \mu), \boldsymbol{P}_{f}^{(j)}(\boldsymbol{z}, \mu)\right) \\
= & \sum_{i=1}^{J(\mu)} w^{(i)}(\mu) \mathcal{N}\left(\boldsymbol{\xi} ; \boldsymbol{m}^{(i)}(\mu), \boldsymbol{P}^{(i)}(\mu)\right)
\end{aligned}
$$

where 


$$
\begin{aligned}
& \boldsymbol{m}_{0}^{(j)}(\mu)=\boldsymbol{m}_{+}^{(j)}(\mu), \boldsymbol{P}_{0}^{(j)}(\mu)=\boldsymbol{P}_{+}^{(j)}(\mu) \\
& {\left[\boldsymbol{m}_{f}^{(j)}(\mu), \boldsymbol{P}_{f}^{(j)}(\mu), q_{f}^{(j)}(\mu)\right]=} \\
& \operatorname{KF} \_\operatorname{UwF}\left(y_{f}(\cdot), \boldsymbol{m}_{+}^{(j)}(\mu), \boldsymbol{P}_{+}^{(j)}(\mu), \boldsymbol{H}_{f}(\cdot), R_{f}, n_{c}\left(\cdot, \boldsymbol{\xi}_{s}\right)\right) \\
& {\left[\boldsymbol{m}_{c}^{(j)}\left(\boldsymbol{y}_{c}, \mu\right), \boldsymbol{P}_{c}^{(j)}\left(\boldsymbol{y}_{c}, \mu\right), q_{c}^{(j)}\left(\boldsymbol{y}_{c}, \mu\right)\right]} \\
& =\mathrm{KF} \_\mathrm{UwC}\left(\boldsymbol{y}_{c}, \boldsymbol{m}_{+}^{(j)}(\mu), \boldsymbol{P}_{+}^{(j)}(\mu), \boldsymbol{H}_{c}, \boldsymbol{R}_{c}\left(\boldsymbol{y}_{p}\right)\right) \\
& {\left[\boldsymbol{m}_{d}^{(j)}(\boldsymbol{z}, \mu), \boldsymbol{P}_{d}^{(j)}(\boldsymbol{z}, \mu), q_{d}^{(j)}\left(y_{d}, \mu\right)\right]=} \\
& \mathrm{KF} \_\mathrm{UwD}\left(y_{d}, \boldsymbol{m}_{c}^{(j)}\left(\boldsymbol{y}_{c}, \mu\right), \boldsymbol{P}_{c}^{(j)}\left(\boldsymbol{y}_{c}, \mu\right), h_{d}(\cdot), \sigma_{d}^{2}\right) \\
& {\left[\boldsymbol{m}_{f}^{(j)}(\boldsymbol{z}, \mu), \boldsymbol{P}_{f}^{(j)}(\boldsymbol{z}, \mu), q_{f}^{(j)}(\boldsymbol{z}, \mu)\right]=} \\
& \mathrm{KF}_{-} \operatorname{UwF}\left(y_{f}(\cdot), \boldsymbol{m}_{d}^{(j)}(\boldsymbol{z}, \mu), \boldsymbol{P}_{d}^{(j)}(\boldsymbol{z}, \mu), \boldsymbol{H}_{f}(\cdot), R_{f}, n_{c}\left(\cdot, \boldsymbol{\xi}_{s}\right)\right) \\
& {\left[w_{0}^{(j)}(\mu), w_{f}^{(j)}(\mu), w_{d}^{(j)}(\boldsymbol{z}, \mu), w_{f}^{(j)}(\boldsymbol{z}, \mu)\right]=} \\
& \text { cmpt_w }\left(w_{+}^{(j)}(\mu), q_{f}^{(j)}(\mu), q_{c}^{(j)}\left(\boldsymbol{y}_{c}, \mu\right), q_{d}^{(j)}\left(y_{d}, \mu\right), q_{f}^{(j)}(z, \mu), p_{D}, c_{f}\right) \\
& \tilde{w}_{d}^{(j)}(\boldsymbol{z}, \mu)=\frac{w_{d}^{(j)}(\boldsymbol{z}, \mu)}{\kappa(\boldsymbol{z})+\sum_{\tilde{\mu}} \sum_{i=1}^{J_{+}(\tilde{\mu})}\left[w_{d}^{(i)}(\boldsymbol{z}, \tilde{\mu})+w_{f}^{(i)}(\boldsymbol{z}, \tilde{\mu})\right]} \\
& \tilde{w}_{f}^{(j)}(\boldsymbol{z}, \mu)=\frac{w_{f}^{(j)}(\boldsymbol{z}, \mu)}{\kappa(\boldsymbol{z})+\sum_{\tilde{\mu}} \sum_{i=1}^{J_{+}(\tilde{\mu})}\left[w_{d}^{(i)}(\boldsymbol{z}, \tilde{\mu})+w_{f}^{(i)}(\boldsymbol{z}, \tilde{\mu})\right]} \\
& \kappa(\boldsymbol{z})=\kappa_{c}\left(\boldsymbol{y}_{c}\right) \kappa_{d}\left(y_{d}\right)
\end{aligned}
$$

\begin{tabular}{|c|c|}
\hline & $\left.\boldsymbol{P}_{f}, q_{f}\right]=\mathrm{KF}_{-} \mathrm{UwF}\left(y_{f}(\cdot), \boldsymbol{m}_{+}, \boldsymbol{P}_{+}, \boldsymbol{H}_{f}(\cdot), R_{f}, n_{c}\right)$ \\
\hline \multicolumn{2}{|c|}{ if $\left|n_{c}\left(\boldsymbol{m}_{+}, \boldsymbol{\xi}_{s}\right)\right|<\mathrm{MDV}$} \\
\hline \multicolumn{2}{|r|}{$\eta_{f}=\boldsymbol{H}_{f}\left(\boldsymbol{m}_{+}\right) \cdot \boldsymbol{m}_{+}$} \\
\hline \multicolumn{2}{|r|}{$S_{f}=\boldsymbol{H}_{f}\left(\boldsymbol{m}_{+}\right) \boldsymbol{P}_{+}\left[\boldsymbol{H}_{f}\left(\boldsymbol{m}_{+}\right)\right]^{\mathrm{T}}+R_{f}$} \\
\hline \multicolumn{2}{|r|}{$\boldsymbol{K}_{f}=\boldsymbol{P}_{+}\left[\boldsymbol{H}_{f}\left(\boldsymbol{m}_{+}\right)\right]^{\mathrm{T}}\left(S_{f}\right)^{-1}$} \\
\hline \multicolumn{2}{|r|}{$\boldsymbol{m}_{f}=\boldsymbol{m}_{+}+\boldsymbol{K}_{f} \cdot\left[y_{f}\left(\boldsymbol{m}_{+}\right)-\eta_{f}\right]$} \\
\hline \multicolumn{2}{|r|}{$\boldsymbol{P}_{f}=\boldsymbol{P}_{+}-\boldsymbol{K}_{f} S_{f}\left[\boldsymbol{K}_{f}\right]^{\mathrm{T}}$} \\
\hline \multicolumn{2}{|r|}{$q_{f}=\mathcal{N}\left(y_{f}\left(\boldsymbol{m}_{+}\right) ; \eta_{f}, S_{f}\right)$} \\
\hline \multicolumn{2}{|c|}{ else } \\
\hline \multicolumn{2}{|r|}{$\boldsymbol{m}_{f}=\emptyset, \boldsymbol{P}_{f}=\emptyset, q_{f}=0$} \\
\hline end & \\
\hline
\end{tabular}

In the above, $\kappa_{c}\left(\boldsymbol{y}_{c}\right)$ and $\kappa_{d}\left(y_{d}\right)$ are the clutter intensity for the position component and Doppler component, respectively. Functions "KF_UwF", "KF_UwC" and "KF_UwD", denoting update operators of the (Extended) Kalman filter using fictitious measurements, converted position measurements, and Doppler measurements, are summarized in Tables II, III and IV, respectively. Function "cmpt_w", used to compute associated weights, is summarized in Table IV.

TABLE II

FUNCTION KF_UwF

In Table II, $\boldsymbol{H}_{f}(\cdot)$ is the Jacobian matrix of the clutter notch with respect to a target state, given in Appendix D. The "if" judgment sentence is based on the following consideration: we only need to preserve components corresponding to targets within the DBZ, thus a component will be updated with the fictitious measurement only when its mean $\boldsymbol{m}_{+}$satisfies $\left|n_{c}\left(\boldsymbol{m}_{+}, \boldsymbol{\xi}_{s}\right)\right|<$ MDV .

TABLE III

FUNCTION KF_UwC

$\left[\boldsymbol{m}_{c}\left(\boldsymbol{y}_{c}\right), \boldsymbol{P}_{c}\left(\boldsymbol{y}_{c}\right), q_{c}\left(\boldsymbol{y}_{c}\right)\right]=\mathrm{KF} \_\mathrm{UwC}\left(\boldsymbol{y}_{c}, \boldsymbol{m}_{+}, \boldsymbol{P}_{+}, \boldsymbol{H}_{c}, \boldsymbol{R}_{c}\left(\boldsymbol{y}_{p}\right)\right)$

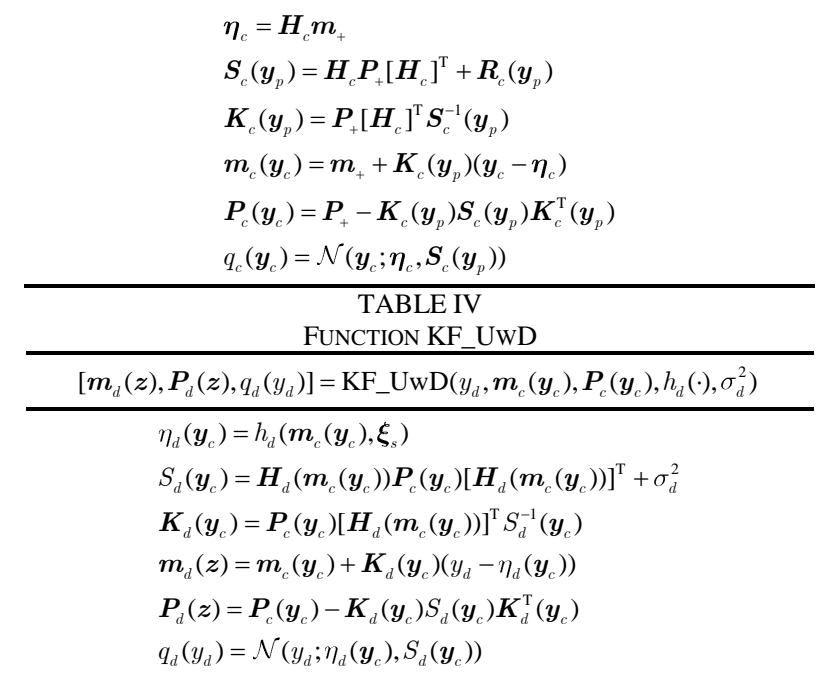

In Table IV, $\boldsymbol{H}_{d}\left(\boldsymbol{m}\left(\boldsymbol{y}_{c}\right)\right)$ is the Jacobian matrix of radial velocity $\dot{r}$ (6) with respect to target state $\xi$ evaluated at $\boldsymbol{m}\left(\boldsymbol{y}_{c}\right)=\left[\begin{array}{llllll}\hat{x} & \hat{y} & \hat{z} & \hat{\dot{x}} & \hat{\dot{y}} & \hat{\dot{z}}\end{array}\right]^{\mathrm{T}}$, calculated as follows

$\boldsymbol{H}_{d}\left(\boldsymbol{m}_{c}\left(\boldsymbol{y}_{c}\right)\right)=\left.\frac{\partial \dot{r}}{\partial \boldsymbol{\xi}}\right|_{\xi=m_{c}\left(y_{c}\right)}=\left[\begin{array}{llllll}h_{1} & h_{2} & h_{3} & h_{4} & h_{5} & h_{6}\end{array}\right]$

where

$h_{1}=\left[\left(\hat{\dot{x}}-\dot{x}_{s}\right)-\hat{\dot{r}} \cos \hat{a} \cos \hat{e}\right] / \hat{r}, h_{2}=\left[\left(\hat{\dot{y}}-\dot{y}_{s}\right)-\hat{\dot{r}} \sin \hat{a} \cos \hat{e}\right] / \hat{r}$, $h_{3}=\left[\left(\hat{\dot{z}}-\dot{z}_{s}\right)-\hat{\dot{r}} \sin \hat{e}\right] / \hat{r}, h_{4}=\cos \hat{a} \cos \hat{e}, \quad h_{5}=\sin \hat{a} \cos \hat{e}$, $h_{6}=\sin \hat{e} \quad, \quad[\hat{r}, \hat{a}, \hat{e}]=\operatorname{Cart} 2 \operatorname{Polar}\left(\boldsymbol{m}\left(\boldsymbol{y}_{c}\right), \boldsymbol{\xi}_{s}\right) \quad$, $\dot{r}=h_{d}\left(\boldsymbol{m}\left(\boldsymbol{y}_{c}\right), \boldsymbol{\xi}_{s}\right)$.

TABLE V FUNCTION CMPT_W

\begin{aligned}${\left[w_{0}, w_{f}, w_{d}(\boldsymbol{z}), w_{f}(\boldsymbol{z})\right] } & =\mathrm{cmpt} \_\mathrm{w}\left(w_{+}, q_{f}, q_{c}\left(\boldsymbol{y}_{c}\right), q_{d}\left(y_{d}\right), q_{f}(\boldsymbol{z}), p_{D}, c_{f}\right) \\$\hline$w_{0} & =\left(1-p_{D}\right) w_{+} \\ w_{f} & =p_{D} c_{f} w_{+} q_{f} \\ w_{d}(\boldsymbol{z}) & =p_{D} w_{+} q_{c}\left(\boldsymbol{y}_{c}\right) q_{d}\left(y_{d}\right) \\ w_{f}(\boldsymbol{z}) & =-c_{f} w_{d}(\boldsymbol{z}) q_{f}(\boldsymbol{z})\end{aligned}$

From the updated intensity (26), the expected number of targets is

$$
\begin{aligned}
\bar{N}= & \sum_{\mu} \sum_{i=1}^{J(\mu)} w^{(i)}(\mu) \\
= & \sum_{\mu} \sum_{j=1}^{J_{+}(\mu)}\left[w_{0}^{(j)}(\mu)+w_{f}^{(j)}(\mu)\right]+ \\
& \sum_{z \in Z} \sum_{\mu} \sum_{j=1}^{J_{+}(\mu)}\left[\tilde{w}_{d}^{(j)}(\boldsymbol{z}, \mu)+\tilde{w}_{f}^{(j)}(\boldsymbol{z}, \mu)\right]
\end{aligned}
$$

Remark: After measurement update, a "pruning-merging" procedure and a multi-target state extraction are required. The "pruning-merging" procedure are the same as those in [27]. For the multi-target state extraction, the cardinality estimate is $\hat{N}$, which is rounded to the nearest integer of $\bar{N}$. The multi-target state estimate $\hat{X}$ is the set of $\hat{N}$ ordered pairs of means and modes with the largest weights. Besides $\hat{X}=\left\{\hat{\boldsymbol{\xi}}_{i}, \hat{\mu}_{i}\right\}, i=1, \cdots,|\hat{X}|$, it is assumed that the multi-target state extraction also outputs associated covariances $\boldsymbol{P}=\left\{\boldsymbol{P}_{i}\right\}, i=1, \cdots,|\hat{X}|$, which is used in the ATI. 


\section{Adaptive track initiation}

To cope with the problem of fixed track initiation, an adaptive track initiation for a GMTI radar is introduced. It includes two steps: first selecting measurements which may be originated from birth targets, and then initializing GM parameters for the births from selected measurements.

1) Selecting measurements originated from birth targets

To realize measurement-driven ATI, we select measurements $Z_{b} \subseteq Z$ originated from birth targets by removing measurements located near the current multi-target state estimate $\hat{X}$ because measurements near $\hat{X}$ are not likely originated from birth targets.

Pseudo-codes for measurement selection are given in Table VI, in which $\hat{X}=\left\{\hat{\boldsymbol{\xi}}_{i}, \hat{\mu}_{i}\right\}$ and $\boldsymbol{P}=\left\{\boldsymbol{P}_{i}\right\}$, obtained from the multi-target state extraction, are the multi-target estimation and associated covariance, $Z_{c}=\left\{\boldsymbol{z}_{c, j}\right\}, j=1, \cdots,\left|Z_{c}\right|$ is a set of converted measurement $\boldsymbol{z}_{c, j}=\left[\boldsymbol{y}_{c, j} ; y_{d, j}\right] \quad$ and $Y_{c}=\left\{\boldsymbol{y}_{c, j}\right\}, j=1, \cdots,\left|Y_{c}\right|$ is a set of converted position measurement $\boldsymbol{y}_{c, j}=\left[x_{m, j}, y_{m, j}, z_{m, j}\right]^{\mathrm{T}}$ with $\left|Y_{c}\right|=\left|Z_{c}\right|$. Gate statistic $d^{(i, j)}$ is assumed to be chi-square distributed with degree of freedom $n_{y}$ with $n_{y}$ being the dimension of position measurement $\boldsymbol{y}_{c, j}$. Hence, threshold $\tau$ is set to be $\tau=\operatorname{chi} 2 \operatorname{inv}\left(p_{G}, n_{y}\right)$, where "chi2inv" denotes the chi-square inverse cumulative distribution function and $p_{G}$ denotes the gate probability.

TABLE VI

PSEUDO-CODES OF SELECTING MEASUREMENTS FOR BIRTH TARGETS

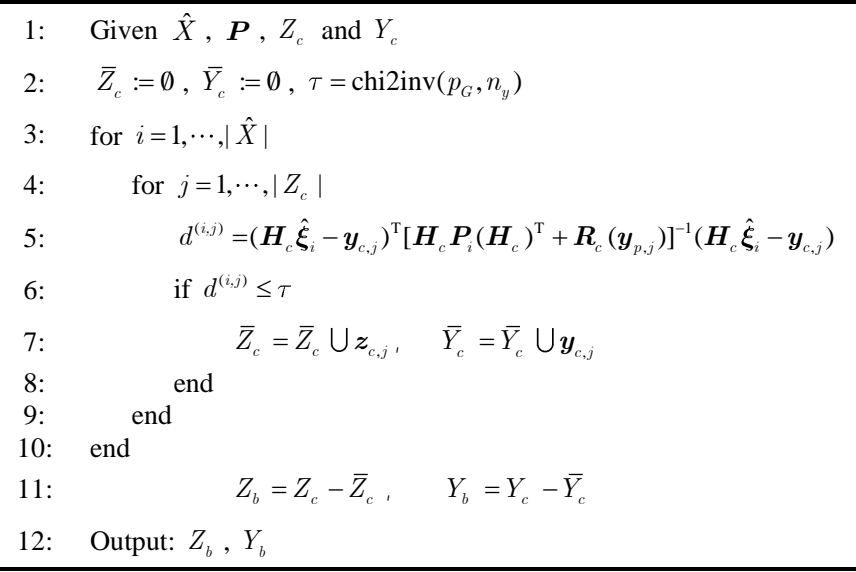

Re $m a$ : The idea of selecting measurements originated from birth targets is inspired by [62], but there exist the following differences. First, the covariance set $\boldsymbol{P}$ is added in Line 1 for calculating gate statistic $d^{(i, j)}$. Second, threshold $\tau$ in Line 2 is calculated by $\tau=\operatorname{chi} 2 \operatorname{inv}\left(p_{G}, n_{y}\right)$, while it is directly set to be $\tau=\sigma_{r}$ in Section IV in [62] with $\sigma_{r}$ being the range error standard deviation. Third, gate statistic in Line 5 is a Mahalanobis distance while it is simply a Euclidean distance in eq. (17) in [62]. Fourth, gate statistic is set to be $d^{(i, j)} \leq \tau$ in Line 6 while it is set to be $d^{(i, j)}>\tau$ (see eq. (18) in [62]), which finally leads to an additional Line 11, where subtraction sign denotes set difference.

\section{2) Initializing associated parameters for the births}

With the selected measurement set $Z_{b}$ or $Y_{b}$, associated parameters (see $w_{\gamma}^{(i)}(\mu), \boldsymbol{m}_{\gamma}^{(i)}(\mu), \boldsymbol{P}_{\gamma}^{(i)}(\mu), p_{\gamma}(\mu)$ and $J_{\gamma}(\mu)$ in Section IV.A, and these parameters will become a prior ones at time $k+1$ ) for the births at time $k$ can be obtained by

$$
\begin{gathered}
J_{\gamma}(\mu)=\left|Z_{b}\right|=\left|Y_{b}\right|, \mu \in \mathbb{U} \\
\boldsymbol{m}_{\gamma}^{(j)}(\mu)=\boldsymbol{m}_{\gamma}^{(j)}, \boldsymbol{P}_{\gamma}^{(j)}(\mu)=\boldsymbol{P}_{\gamma}^{(j)}, j=1, \cdots,\left|Z_{b}\right|, \mu \in \mathbb{U}
\end{gathered}
$$

In addition, the distribution of modes at birth $p_{\gamma}(\mu)$ and $w_{\gamma}^{(i)}(\mu) \quad$ can be specified by users. In (39), $\boldsymbol{m}_{\gamma}^{(j)}=\left[x_{\gamma}^{(j)}, y_{\gamma}^{(j)}, z_{\gamma}^{(j)}, \dot{x}_{\gamma}^{(j)}, \dot{y}_{\gamma}^{(j)}, \dot{z}_{\gamma}^{(j)}\right]^{\mathrm{T}}$ and $\boldsymbol{P}_{\gamma}^{(j)}=\operatorname{blkdiag}\left(\boldsymbol{P}_{\gamma, p}^{(j)}, \boldsymbol{P}_{\gamma, v}^{(j)}\right)$ can be calculated as follows.

Based on $Z_{b}=\left\{\boldsymbol{z}_{c, j}\right\}$ and $Y_{b}=\left\{\boldsymbol{y}_{c, j}\right\}, j=1, \cdots,\left|Z_{b}\right|$, where $\boldsymbol{z}_{c, j}=\left[\boldsymbol{y}_{c, j} ; y_{d, j}\right]$ and $\boldsymbol{y}_{c, j}=\left[x_{m, j}, y_{m, j}, z_{m, j}\right]^{\mathrm{T}}$, the position component of $\boldsymbol{m}_{\gamma}^{(j)}$ is given by $\left[x_{\gamma}^{(j)}, y_{\gamma}^{(j)}, z_{\gamma}^{(j)}\right]^{\mathrm{T}}=\boldsymbol{y}_{c, j}$, which is obtained from $\boldsymbol{y}_{p, j}$ via function "Polar2Cart" in Appendix B, and the position part $\boldsymbol{P}_{\gamma, p}^{(j)}$ of covariance $\boldsymbol{P}_{\gamma}^{(j)}$ is given by (53) in Appendix C, i.e., $\boldsymbol{P}_{\gamma, p}^{(j)}=\boldsymbol{R}_{c}\left(\boldsymbol{y}_{p, j}\right)$. In a word, these quantities are obtained from measurement transformation in Section IV.B.1. Now, the remaining problems are calculating the velocity parts of $\boldsymbol{m}_{\gamma}^{(j)}$ and $\boldsymbol{P}_{\gamma}^{(j)}$, i.e., $\left[\dot{x}_{\gamma}^{(j)}, \dot{y}_{\gamma}^{(j)}, \dot{z}_{\gamma}^{(j)}\right]^{\mathrm{T}}$ and $\boldsymbol{P}_{\gamma, v}^{(j)}$.

From (4) and (6), the measurement equation for the Doppler component can be written in terms of a GM component as

$$
y_{d, j}=\boldsymbol{H}_{v}^{(j)}\left(\dot{\boldsymbol{m}}_{\gamma}^{(j)}-\dot{\boldsymbol{\xi}}_{s}\right)+n_{d, j}
$$

where $\boldsymbol{H}_{v}^{(j)}=\left[\cos a^{(j)} \cos e^{(j)}, \sin a^{(j)} \cos e^{(j)}, \sin e^{(j)}\right]$ and $\left[\sim, a^{(j)}, e^{(j)}\right]=\operatorname{Cart} 2 \operatorname{Polar}\left(\boldsymbol{m}_{\gamma}^{(j)}, \boldsymbol{\xi}_{s}\right) ; \dot{\boldsymbol{m}}_{\gamma}^{(j)}$ and $\dot{\boldsymbol{\xi}}_{s}$ are the velocity parts of component $\boldsymbol{m}_{\gamma}^{(j)}$ and sensor state $\boldsymbol{\xi}_{s}$, respectively. Assume that $n_{d, j} \sim \mathcal{N}\left(n_{d, j} ; 0, \sigma_{d}^{2}\right)$, and $\dot{\boldsymbol{m}}_{\gamma}^{(j)} \sim \mathcal{N}\left(\dot{\boldsymbol{m}}_{\gamma}^{(j)} ; \boldsymbol{O}, \boldsymbol{R}_{v}\right)$ where $\boldsymbol{R}_{v}=\operatorname{blkdiag}\left(\sigma_{v}^{2}, \sigma_{v}^{2}, \sigma_{v}^{2}\right)$ with $\sigma_{v}$ being a prior speed standard deviation. Then, the linear minimum mean square error (LMMSE) estimate and the corresponding covariance become [22]

$$
\begin{aligned}
& {\left[\dot{x}_{\gamma}^{(j)}, \dot{y}_{\gamma}^{(j)}, \dot{z}_{\gamma}^{(j)}\right]^{\mathrm{T}} } \\
= & \boldsymbol{R}_{v}\left(\boldsymbol{H}_{v}^{(j)}\right)^{\mathrm{T}}\left[\boldsymbol{H}_{v}^{(j)} \boldsymbol{R}_{v}\left(\boldsymbol{H}_{v}^{(j)}\right)^{\mathrm{T}}+\sigma_{d}^{2}\right]^{-1} y_{d, j}+\left[\dot{x}_{s}, \dot{y}_{s}, \dot{z}_{s}\right]^{\mathrm{T}} \\
= & \beta\left(\boldsymbol{H}_{v}^{(j)}\right)^{\mathrm{T}} y_{d, j}+\left[\dot{x}_{s}, \dot{y}_{s}, \dot{z}_{s}\right]^{\mathrm{T}} \\
\boldsymbol{P}_{\gamma, v}^{(j)}= & \boldsymbol{R}_{v}-\boldsymbol{R}_{v}\left(\boldsymbol{H}_{v}^{(j)}\right)^{\mathrm{T}}\left[\boldsymbol{H}_{v}^{(j)} \boldsymbol{R}_{v}\left(\boldsymbol{H}_{v}^{(j)}\right)^{\mathrm{T}}+\sigma_{d}^{2}\right]^{-1} \boldsymbol{H}_{v}^{(j)} \boldsymbol{R}_{v}^{\mathrm{T}} \\
= & \sigma_{v}^{2}\left[\begin{array}{lll}
P_{11} & P_{12} & P_{13} \\
P_{12} & P_{22} & P_{23} \\
P_{13} & P_{23} & P_{33}
\end{array}\right]
\end{aligned}
$$

where $\beta=\sigma_{v}^{2}\left(\sigma_{v}^{2}+\sigma_{d}^{2}\right)^{-1}$, and

$$
\begin{aligned}
& P_{11}=1-\beta \cos ^{2} a^{(j)} \cos ^{2} e^{(j)} \\
& P_{12}=-\beta \sin a^{(j)} \cos a^{(j)} \cos ^{2} e^{(j)}
\end{aligned}
$$




$$
\begin{aligned}
& P_{13}=-\beta \cos a^{(j)} \sin e^{(j)} \cos e^{(j)} \\
& P_{22}=1-\beta \sin ^{2} a^{(j)} \cos ^{2} e^{(j)} \\
& P_{23}=-\beta \sin a^{(j)} \sin e^{(j)} \cos e^{(j)} \\
& P_{33}=1-\beta \sin ^{2} e^{(j)}
\end{aligned}
$$

At this point, the ATI task is completed.

\section{Extension to track label propagation}

The above MM-PHD filter with the Doppler, MDV, and ATI can be further extended to propagate track continuity, by incorporating a unique track label [3][63]. More specifically, a unique label $\ell^{(i)}(\mu)$ is assigned to each GM component $\left(w^{(i)}(\mu), \boldsymbol{m}^{(i)}(\mu), \boldsymbol{P}^{(i)}(\mu)\right)$. The collection of quadruples $\mathcal{T}=\left\{\left(\ell^{(i)}(\mu), w^{(i)}(\mu), \boldsymbol{m}^{(i)}(\mu), \boldsymbol{P}^{(i)}(\mu)\right)\right\}_{i=1, \cdots, J}^{\mu \in \mathbb{U}}$ is referred to as a track table. To evaluate the overall GMTI tracking performance, a simple scheme is presented as follows.

Prediction: if the prior track table $\mathcal{T}^{\prime}$ at time $k-1$ is the union of $\left\{\left(\ell_{S}^{(i)}\left(\mu^{\prime}\right), w_{S}^{(i)}\left(\mu^{\prime}\right), \boldsymbol{m}_{S}^{(i)}\left(\mu^{\prime}\right), \boldsymbol{P}_{S}^{(i)}\left(\mu^{\prime}\right)\right)\right\}_{i=1, \cdots, J_{S}^{\prime}\left(\mu^{\prime}\right)}^{\mu^{\prime}, \mathbb{U}}$ and $\left\{\left(\ell_{\gamma}^{(i)}\left(\mu^{\prime}\right), w_{\gamma}^{(i)}\left(\mu^{\prime}\right), \boldsymbol{m}_{\gamma}^{(i)}\left(\mu^{\prime}\right), \boldsymbol{P}_{\gamma}^{(i)}\left(\mu^{\prime}\right)\right)\right\}_{i=1, \cdots, J_{\gamma}^{\prime}\left(\mu^{\prime}\right)}^{\mu^{\prime}, \mathbb{U}} \quad$, then the predicted track table $\mathcal{T}_{+}$to time $k$ is

$$
\begin{gathered}
\mathcal{T}_{+}=\left\{\left(\ell_{S,+}^{(i)}\left(\mu, \mu^{\prime}\right), w_{S,+}^{(i)}\left(\mu, \mu^{\prime}\right), \boldsymbol{m}_{S,+}^{(i)}\left(\mu, \mu^{\prime}\right), \boldsymbol{P}_{S,+}^{(i)}\left(\mu, \mu^{\prime}\right)\right)\right\}_{i=1, \cdots, J_{S}^{\prime}\left(\mu^{\prime}\right)}^{\mu^{\prime} \in \mathbb{U}, \mu \in \mathbb{U}} \\
\bigcup\left\{\left(\ell_{\gamma,+}^{(i)}\left(\mu, \mu^{\prime}\right), w_{\gamma,+}^{(i)}\left(\mu, \mu^{\prime}\right), \boldsymbol{m}_{\gamma,+}^{(i)}\left(\mu, \mu^{\prime}\right), \boldsymbol{P}_{\gamma,+}^{(i)}\left(\mu, \mu^{\prime}\right)\right)\right\}_{i=1, \cdots, J_{\gamma}^{\prime}\left(\mu^{\prime}\right)}^{\mu^{\prime} \in \mathbb{U}, \mu \mathbb{U}^{\prime}}
\end{gathered}
$$

where $w_{S,+}^{(i)}\left(\mu, \mu^{\prime}\right), \boldsymbol{m}_{S,+}^{(i)}\left(\mu, \mu^{\prime}\right), \boldsymbol{P}_{S,+}^{(i)}\left(\mu, \mu^{\prime}\right), w_{\gamma,+}^{(i)}\left(\mu, \mu^{\prime}\right)$, $\boldsymbol{m}_{\gamma,+}^{(i)}\left(\mu, \mu^{\prime}\right), \boldsymbol{P}_{\gamma,+}^{(i)}\left(\mu, \mu^{\prime}\right)$ are given in Section IV.A, and $\ell_{S,+}^{(i)}\left(\mu, \mu^{\prime}\right)=\ell_{S}^{(i)}\left(\mu^{\prime}\right), \ell_{\gamma,+}^{(i)}\left(\mu, \mu^{\prime}\right)=\ell_{\gamma}^{(i)}\left(\mu^{\prime}\right)$. Hence, predicted components inherit from their previous labels.

Update: if the predicted track table to time $k$ is $\mathcal{T}_{+}=\left\{\left(\ell_{+}^{(i)}(\mu), w_{+}^{(i)}(\mu), \boldsymbol{m}_{+}^{(i)}(\mu), \boldsymbol{P}_{+}^{(i)}(\mu)\right)\right\}_{i=1, \cdots, J_{+}(\mu)}^{\mu \in \mathbb{U}}$, then the updated track table at time $k$ is

$$
\begin{aligned}
\mathcal{T}= & \left\{\left(\ell_{0}^{(i)}(\mu), w_{0}^{(i)}(\mu), \boldsymbol{m}_{0}^{(i)}(\mu), \boldsymbol{P}_{0}^{(i)}(\mu)\right)\right\}_{i=1, \cdots, J_{+}(\mu)}^{\mu \in \mathbb{U}} \cup \\
& \left\{\left(\ell_{f}^{(i)}(\mu), w_{f}^{(i)}(\mu), \boldsymbol{m}_{f}^{(i)}(\mu), \boldsymbol{P}_{f}^{(i)}(\mu)\right)\right\}_{i=1, \cdots, J_{+}(\mu)}^{\mu \in \mathbb{U}} \cup \\
& \left\{\left(\ell_{d}^{(i)}(\boldsymbol{z}, \mu), \tilde{w}_{d}^{(i)}(\boldsymbol{z}, \mu), \boldsymbol{m}_{d}^{(i)}(\boldsymbol{z}, \mu), \boldsymbol{P}_{d}^{(i)}(\boldsymbol{z}, \mu)\right)\right\}_{i=1, \cdots, J_{+}(\mu), z \in Z}^{\mu \in \mathbb{U}} U \\
& \left\{\left(\ell_{f}^{(i)}(\boldsymbol{z}, \mu), \tilde{w}_{f}^{(i)}(\boldsymbol{z}, \mu), \boldsymbol{m}_{f}^{(i)}(\boldsymbol{z}, \mu), \boldsymbol{P}_{f}^{(i)}(\boldsymbol{z}, \mu)\right)\right\}_{i=1, \cdots, J_{+}(\mu), \boldsymbol{z} \in Z}^{\mu \in \mathbb{U}}
\end{aligned}
$$

where weights, means, and covariances are given in Section IV.B, and $\ell_{0}^{(i)}(\mu)=\ell_{f}^{(i)}(\mu)=\ell_{d}^{(i)}(z, \mu)=\ell_{f}^{(i)}(z, \mu)=\ell_{+}^{(i)}(\mu)$. Hence, $2 \times(1+|Z|)$ updated Gaussians are assigned the same label as that of their predecessor $\ell_{+}^{(i)}(\mu)$.

Pruning-merging and multi-target state extraction: both steps are added appropriate label processing. For example, in the pruning-merging procedure, the component with the largest weight is appointed as the predecessor.

Adaptive track initiation: during initiation in Section IV.C, new labels are assigned for birth components, i.e., the birth track table at time $k$ is $\mathcal{T}_{\gamma}=\left\{\left(\ell_{\gamma}^{(i)}(\mu), w_{\gamma}^{(i)}(\mu), \boldsymbol{m}_{\gamma}^{(i)}(\mu), \boldsymbol{P}_{\gamma}^{(i)}(\mu)\right)\right\}_{i=1, \cdots, J_{\gamma}(\mu)}^{\mu \in \mathbb{U}} \quad$, where $\ell_{\gamma}^{(i)}(\mu)=(k, i), \mu \in \mathbb{U}$, to guarantee the uniqueness of track labels per model. It is noted again that, unlike conventional methods [3], new labels for birth components are assigned in the initiation, rather than in the prediction.
$R e m a$ Frok the sake of brevity, the proposed MM-PHD filter with the Doppler, MDV, ATI, and TLP is called the MMPHD_DBZ tracker. Note that the PHD filter with the Doppler and MDV in [4] is a special case of the proposed tracker when $|\mathbb{U}|=1$, and the MM-PHD filter in [37] is also a special version of the proposed tracker when MDV $=0$. Nevertheless, both special versions don't have the ATI and TLP capabilities. To fairly compare the overall performance, the ATI and TLP functions proposed in this paper are additionally enhanced for both special versions. Hereafter, the augmented approach from [4] is called the PHD_DBZ tracker. Besides, unless explicitly stated, the MM-PHD filter has ATI and TLP functions by default.

\section{Simulation AnAlysis}

For illustration purposes, we consider an example of tracking 3 targets in the 2-dimensional $x-y$ plane with measurements from a moving GMTI sensor, although the proposed tracker is developed in the general 3-dimensional coordinates. It is assumed that each target is moving and mode space $\mathbb{U}$ includes three types of motion models: constant velocity (CV), right turn (coordinated turn with a $3^{\circ}$ angle), and left turn (coordinated turn with a $-3^{\circ}$ angle), which can be expressed as

$$
\boldsymbol{\xi}=\boldsymbol{F}(\mu) \boldsymbol{\xi}^{\prime}+\boldsymbol{v}
$$

where the target state becomes $\boldsymbol{\xi}^{\prime}=\left[\begin{array}{llll}x & y & \dot{x} & \dot{y}\end{array}\right]^{\mathrm{T}}$, the state transition matrices for these three models can be obtained by substituting $№=0$ (for $\mu=1$ ), №= $3 \pi / 180$ (for $\mu=2$ ) and №= $-3 \pi / 180$ (for $\mu=3$ ), respectively, in

$$
\begin{aligned}
\boldsymbol{F}(\mu) & =\boldsymbol{F}(\omega) \\
& =\left[\begin{array}{cccc}
1 & 0 & \sin \left(\omega T_{s}\right) / \omega & -\left(1-\cos \left(\omega T_{s}\right)\right) / \omega \\
0 & 1 & \left(1-\cos \left(\omega T_{s}\right)\right) / \omega & \sin \left(\omega T_{s}\right) / \omega \\
0 & 0 & \cos \left(\omega T_{s}\right) & -\sin \left(\omega T_{s}\right) \\
0 & 0 & \sin \left(\omega T_{s}\right) & \cos \left(\omega T_{s}\right)
\end{array}\right]
\end{aligned}
$$

in which $T_{s}=1 \mathrm{~s}$ is the time interval, and $\boldsymbol{v}$ is a zero-mean white Gaussian process noise with covariance

$$
\boldsymbol{Q}(\mu)=\left[\begin{array}{cc}
T_{s}^{4} / 4 & T_{s}^{3} / 2 \\
T_{s}^{3} / 2 & T_{s}^{2}
\end{array}\right] \otimes \operatorname{diag}\left(\sigma_{x}^{2}, \sigma_{y}^{2}\right), \mu=1,2,3
$$

with $\sigma_{x}^{2}=1 \mathrm{~m} / \mathrm{s}^{2}$ and $\sigma_{y}^{2}=1 \mathrm{~m} / \mathrm{s}^{2}$ being variances of the process noise modeling the acceleration along $x$ and $y$ axes, respectively. Targets are assumed to switch between motion models according to the Markov transition probability matrix (TPM)

$$
[\tau]=\left[\begin{array}{ccc}
0.9 & 0.05 & 0.05 \\
0.05 & 0.9 & 0.05 \\
0.05 & 0.05 & 0.9
\end{array}\right]
$$

Each target has survival probability $p_{S}=0.99$, and it is detected with probability $p_{D}=0.98$. Besides, measurement error standard deviations are $\sigma_{r}=10 \mathrm{~m}, \sigma_{a}=1^{\circ}$ and $\sigma_{d}=0.5 \mathrm{~m} / \mathrm{s}$. Clutters are assumed to be Poison distributed in number with mean $\lambda_{c}=20$, and to be uniformly distributed in the detection region, which is set by the distance range $[0$, $2000](\mathrm{m})$ and angle range $[-\pi, \pi](\mathrm{rad})$. They are modeled as 
$\kappa_{c}(\cdot)=\lambda_{c} p_{c}(\cdot) \quad$ for the position component and $\kappa_{d}(\cdot)=1 /\left(2 v_{\max }\right)$ for the Doppler component, where $p_{c}(\cdot)=1 / V=1 /\left(\pi \times 2000^{2}\right)$ is the clutter spatial distribution, $V$ represents circle area, and $v_{\max }=15 \mathrm{~m} / \mathrm{s}$ is the maximum speed that the sensor can detect.

In the MM-PHD_DBZ tracker, pruning and merging of Gaussian components are performed using a truncation threshold of $10^{-6}$, a merging threshold of $4 \mathrm{~m}$, and a maximum of 100 Gaussian components. For adaptive track initiation, gate probability is $p_{G}=0.999$, a prior speed standard deviation $\sigma_{v}$ is set to be $\sigma_{v}=15 \mathrm{~m} / \mathrm{s}$, the distribution $p_{\gamma}(\mu)$ of models at birth is taken as $p_{\gamma}(1)=0.8, p_{\gamma}(2)=0.1, p_{\gamma}(3)=0.1$, and $w_{\gamma}^{(i)}(\mu)=0.001$ for $i=1, \cdots,\left|Z_{b}\right|, \mu \in \mathbb{U}$. Because the PHD_DBZ and MM-PHD trackers are the special cases of the MM-PHD_DBZ tracker, their common parameters are the same as those of the proposed tracker.

Fig. 4 gives the trajectories of the sensor and targets, where the sensor is traveling along a circle at a constant speed with initial state $\left[\begin{array}{llll}650 \mathrm{~m} & -160 \mathrm{~m} \quad 10 \mathrm{~m} / \mathrm{s} \quad 0 \mathrm{~m} / \mathrm{s}\end{array}\right]^{\mathrm{T}}$ and constant angular (turn) rate $0.063 \mathrm{rad} / \mathrm{s}$, and motion model parameters for these three targets are given in Table VII with

$$
\begin{gathered}
\boldsymbol{x}_{1,0}=\left[\begin{array}{llll}
-400 \mathrm{~m} & 100 \mathrm{~m} & 12 \mathrm{~m} / \mathrm{s} & -4 \mathrm{~m} / \mathrm{s}
\end{array}\right]^{\mathrm{T}} \\
\boldsymbol{x}_{2,0}=\left[\begin{array}{llll}
-400 \mathrm{~m} & 400 \mathrm{~m} & 10 \mathrm{~m} / \mathrm{s} & -5 \mathrm{~m} / \mathrm{s}
\end{array}\right]^{\mathrm{T}} \\
\boldsymbol{x}_{3,0}=\left[\begin{array}{llll}
-200 \mathrm{~m} & -600 \mathrm{~m} & 12 \mathrm{~m} / \mathrm{s} & 3 \mathrm{~m} / \mathrm{s}
\end{array}\right]^{\mathrm{T}} . \\
\text { TABLE VII } \\
\text { MOTION MODEL PARAMETERS FOR 3 TARGETS }
\end{gathered}
$$

\begin{tabular}{ccccc}
\hline \hline Target & Initial state & $\mu=1$ & $\mu=2$ & $\mu=3$ \\
\hline 1 & $\boldsymbol{x}_{1,0}$ & $\begin{array}{c}1 \mathrm{~s}-35 \mathrm{~s}, \\
\text { 1s-100s }\end{array}$ & $51 \mathrm{~s}-60 \mathrm{~s}$ & $36 \mathrm{~s}-50 \mathrm{~s}$ \\
2 & $\boldsymbol{x}_{2,0}$ & $\begin{array}{c}1 \mathrm{~s}-40 \mathrm{~s}, \\
76 \mathrm{~s}-100 \mathrm{~s}\end{array}$ & & $41 \mathrm{~s}-75 \mathrm{~s}$ \\
3 & $\boldsymbol{x}_{3,0}$ & $1 \mathrm{~s}-20 \mathrm{~s}$, & $21 \mathrm{~s}-42 \mathrm{~s}$ & \\
\end{tabular}

In this configuration, the true radial speeds (Dopplers) of three targets and the respective DBZs versus time are shown in Fig. 5. It shows that the time when targets are inside the DBZs is $46 \mathrm{~s}-71 \mathrm{~s}$ for target $1,54 \mathrm{~s}-61 \mathrm{~s}$ for target 2 , and $72 \mathrm{~s}-76 \mathrm{~s}$ for target 3 when $\mathrm{MDV}=3 \mathrm{~m} / \mathrm{s}$, while it is $44 \mathrm{~s}-77 \mathrm{~s}$ for target $1,51 \mathrm{~s}-$ $64 \mathrm{~s}$ for target 2 , and $71 \mathrm{~s}-78 \mathrm{~s}$ for target 3 when $\mathrm{MDV}=5$. Notice that during DBZ masking, target 1 makes a right turn and a left turn, target 2 is making a right turn, and target 3 is making a CV motion. Note also that target 1 is masked up to 34 time steps when $\mathrm{MDV}=5 \mathrm{~m} / \mathrm{s}$.

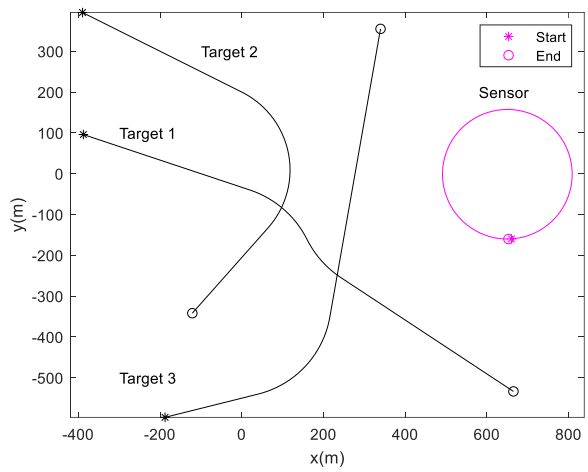

Fig.4. Sensor-to-target geometry.
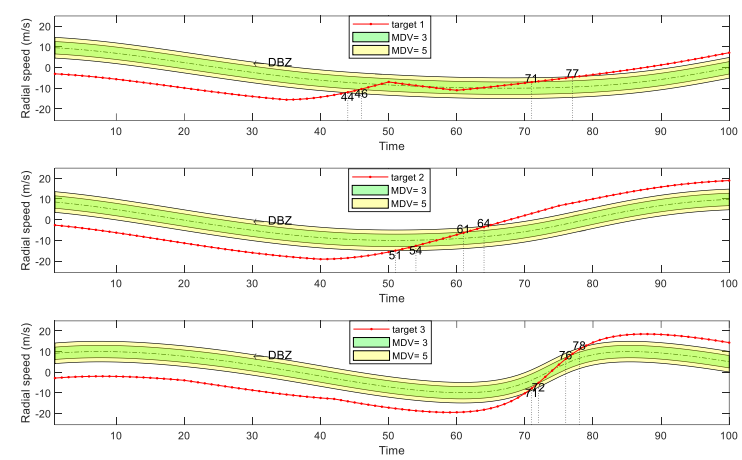

Fig.5. Three targets' Dopplers and the corresponding DBZs versus time when $\mathrm{MDV}=3 \mathrm{~m} / \mathrm{s}$ or $\mathrm{MDV}=5 \mathrm{~m} / \mathrm{s}$.

A typical single-run result when $\mathrm{MDV}=3 \mathrm{~m} / \mathrm{s}$ is given in Fig. 6 , showing the true trajectories and estimated tracks from different trackers. Fig.6(a) is obtained by the standard MMPHD filter [37] augmented with additional TLP. But the augmented tracker is limited to the fixed birth model, set as follows

$$
\begin{aligned}
& \boldsymbol{m}_{\gamma}^{(1)}=\left[\begin{array}{llll}
-400 \mathrm{~m} & 100 \mathrm{~m} & 0 \mathrm{~m} / \mathrm{s} & 0 \mathrm{~m} / \mathrm{s}
\end{array}\right]^{\mathrm{T}} \\
& \boldsymbol{m}_{\gamma}^{(2)}=\left[\begin{array}{llll}
-400 \mathrm{~m} & 400 \mathrm{~m} & 0 \mathrm{~m} / \mathrm{s} & 0 \mathrm{~m} / \mathrm{s}
\end{array}\right]^{\mathrm{T}} \\
& \boldsymbol{m}_{\gamma}^{(3)}=\left[\begin{array}{llll}
-200 \mathrm{~m} & -600 \mathrm{~m} & 0 \mathrm{~m} / \mathrm{s} & 0 \mathrm{~m} / \mathrm{s}
\end{array}\right]^{\mathrm{T}} \\
& \boldsymbol{P}_{\gamma}^{(j)}=\operatorname{blkdiag}\left(10^{2}, 10^{2}, 10^{2}, 10^{2}\right), j=1, \cdots, 3 \\
& p_{\gamma}(1)=0.8, p_{\gamma}(2)=0.1, p_{\gamma}(3)=0.1
\end{aligned}
$$

and $w_{\gamma}^{(j)}(\mu)=0.001, j=1, \cdots, 3, \mu \in \mathbb{U}$. It can be seen that the tracker successfully initiates since it is given by a preset and correct birth model, but it produces target missing once targets enter into the DBZ, even for target 3 , the number of consecutive missed detections caused by the DBZ is only 5 .

Fig.6(b) is obtained by the standard MM-PHD filter [37] augmented with additional TLP and ATI. This augmented tracker seems to have succeeded in tracking targets again after DBZ masking. Nevertheless, its success comes from ATI. Hence, the track re-initiation occurs after targets leave outside the DBZ, which is intuitively reflected in the label switching since different track labels are indicated by different colors. Fig.6(c) is obtained by the PHD_DBZ tracker, which has a certain tracking ability for the targets masked by the DBZ, such as target 3 , since target 3 performs a $\mathrm{CV}$ motion during the masking. Fig.6(d) is obtained by the proposed MM-PHD_DBZ tracker, which can track all the targets without the occurrence of label switching, even targets are maneuvering during the 
masking, e.g., targets 1 and 2 .

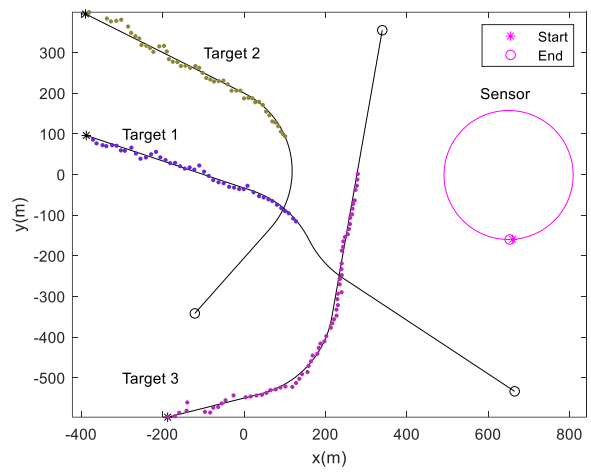

(a) MM-PHD filter augmented with TLP

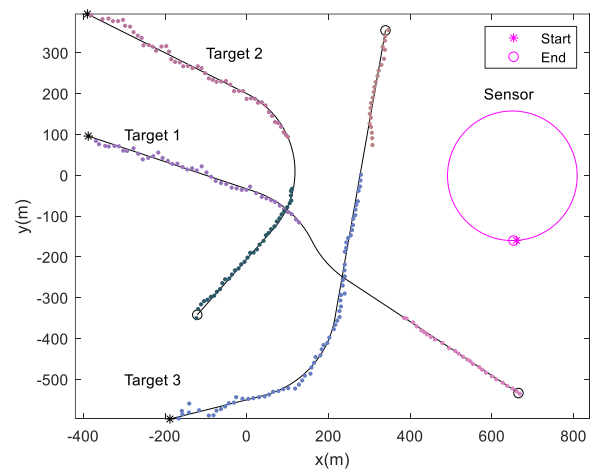

(b) MM-PHD filter augmented with TLP and ATI

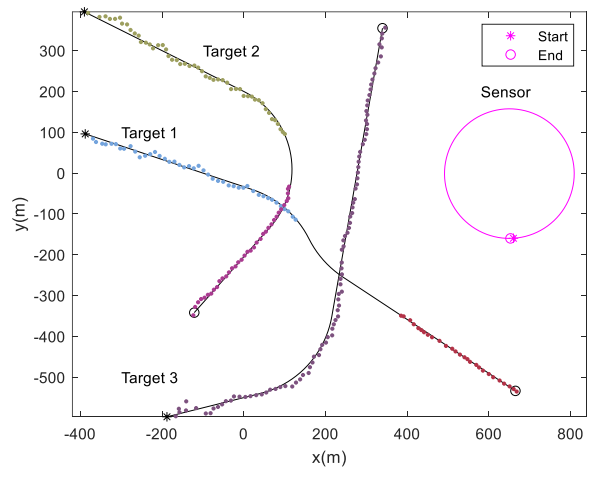

(c) PHD_DBZ

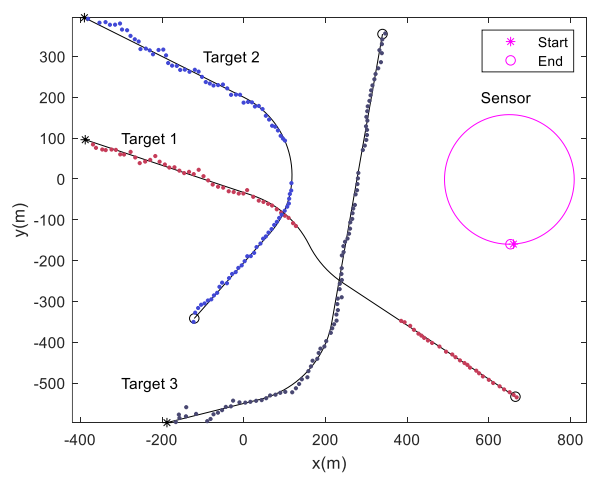

(d) MM-PHD_DBZ

Fig.6. True and estimated tracks from different trackers (MDV=3m/s).

In a word, the above results confirm that the proposed tracker can automatically initiate and maintain the consistency of track labels between before targets entering into and after leaving outside the DBZs. Nevertheless, note that all trackers, including the proposed tracker, cannot extract target states during DBZ masking, due to the inherent mechanism of the PHD-like filters: they are seriously affected by missed detections. The fictitious measurement helps to avoid the deletion of corresponding GM components, but it can't increase their weights to extract the state. After all, the fictitious measurement is not an actual measurement.

Fig.7 gives the performances of different trackers through 100 Monte Carlo runs. In this figure, performance metrics called the optimal sub-pattern assignment (OSPA) for tracks (OSPA-T) [55] and OSPA on OSPA $\left(\mathrm{OSPA}^{(2)}\right)$ [54] are used. The common order and cut-off (see [64] for the meaning of these parameters) are set to be $p=1$ and $c=50 \mathrm{~m}$. Moreover, for the OSPA-T, an additional parameter is set to be $\alpha=50 \mathrm{~m}$ (see eq. (7) in [55] for the meaning of this parameter). For the $\mathrm{OSPA}^{(2)}$, an additional $L_{w}$ (the size of the moving window) is varied. Note that OSPA ${ }^{(2)}$ reduces to the well-known OSPA [64] when $L_{w}=1$.

As shown in Fig.7(a)-(c), although the OSPA ${ }^{(2)}$ incorporates labeling errors, no matter how $L_{w}$ changes, there is no obvious difference in $\mathrm{OSPA}^{(2)}$ of different trackers, which shows that the OSPA $^{(2)}$ cannot reflect the change of track labels. Therefore, it cannot capture label errors caused by label switching due to the DBZ masking. However, this problem can be resolved with OSPA-T.

Let's see Fig.7(a) and Fig.7(d) and compare the OPSA-T with OSPA. For all trackers, their OSPA-T cardinality (Card) components are identical to their respective OSPA Cards. The main difference between the OPSA-T and OSPA is reflected in the localization (Loc) component. For the OSPA, the OSPA Locs of different trackers are very similar since the OSPA cannot capture label errors. On the other hand, for the OSPA-T, the OSPA Locs of different trackers are very different after $61 \mathrm{~s}$. Note that at 61s, target 2 starts to leave outside the DBZ first. Moreover, since OSPA-T Loc consists of the OSPA Loc and label errors [55], the OSPA-T Loc of the MM-PHD_DBZ tracker is slightly larger than its OSPA Loc after $61 \mathrm{~s}$, which means that for the MM-PHD_DBZ tracker, the labels may switch, but the switching probability is low. However, for the PHD_DBZ and especially MM-PHD tracker, their OSPA-T Locs are larger than their respective OSPA Locs, which means that their label switching probabilities are very high. In fact, for the MM-PHD tracker, its estimated labels are switched with probability 1 . In a word, these results indicate that the OSPA-T can capture label errors caused by label switching while the $\mathrm{OSPA}^{(2)}$ (and OSPA) can't do.

Examine Fig.7(d) more closely. As shown by the OSPA-T Card component, during 46s-53s when only target 1 is masked by the DBZ, all of the trackers fail to extract target states. Hence, the OSPA-T Cards increase to about 16, which is approximately cut-off value 50 divided by the number 3 of targets. During 54s61s when both targets 1 and 2 are masked, the OSPA-T Cards further increase to about 32, which is 16 per target times 2 . During $62 \mathrm{~s}-71 \mathrm{~s}$ when target 2 flies outside the DBZ and only target 1 is masked, the OSPA-T Cards fall back to about 16 . During $72 \mathrm{~s}-76 \mathrm{~s}$ there exists a fluctuation in the OSPA-T Cards, since target 1 begins to leave outside the DBZ and target 3 begins to enter into the DBZ at 72s. Once targets are outside the DBZ (e.g., $2 \mathrm{~s}-45 \mathrm{~s}$ and $77 \mathrm{~s}-100 \mathrm{~s}$ ), all of the trackers can 
estimate accurately the number of targets, which means that the ATI is effective.

Nevertheless, the OSPA-T Card cannot reflect the track switching, which can be indicated by the OSPA-T Loc component. From the perspective of the OSPA-T Loc in Fig.7(d), during $61 \mathrm{~s}-71 \mathrm{~s}$ when target 2 leaves outside the DBZ, the MM-PHD tracker will output a changed track label for this target. Hence, its OSPA-T Loc increase to about 16, which is slightly larger than that of the PHD_DBZ tracker. This phenomenon indicates that the MM-PHD tracker has a higher probability of label switching than the PHD_DBZ tracker. However, the OSPA-T Loc of the proposed tracker maintains a level comparable to its previous level, which indicates the proposed tracker can track targets without the occurrence of label switching with a high probability.

Similar analyses can be followed and similar conclusions can be obtained at subsequent times. During 72s-76s when target 1 leaves outside the DBZ, the OSPA-T Loc of the MM-PHD tracker increases to about 32, that of the PHD DBZ tracker increases with its approximately equal increment during $61 \mathrm{~s}-$ $71 \mathrm{~s}$, while that of the proposed tracker increase slightly since target 1 is obscured for too long (reaching 26s, 46s-71s). After target 3 flies outside the DBZ (i.e., after 77s), the OSPA-T Loc of the MM-PHD tracker increases to the cut off even target 3 is masked for a short time (only 5 time steps, $72 \mathrm{~s}-76 \mathrm{~s}$ ), while those of other two trackers maintain the unchanged since target 3 makes a non-maneuvering motion.

Finally, in terms of the overall OSPA-T distance, the proposed tracker outperforms the PHD_DBZ tracker, which is further superior to the MM-PHD tracker.
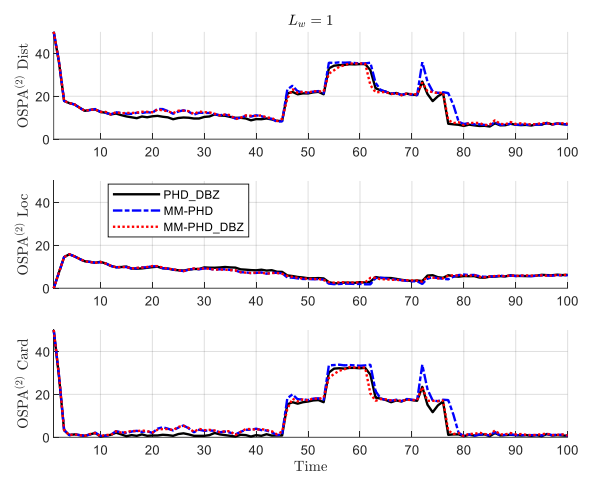

(a) $\operatorname{OSPA}$ or $\operatorname{OSPA}^{(2)}\left(L_{w}=1\right)$
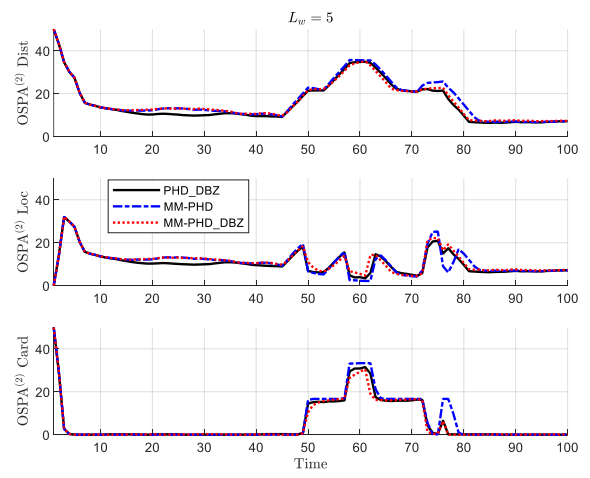

(b) $\operatorname{OSPA}^{(2)}\left(L_{w}=5\right)$
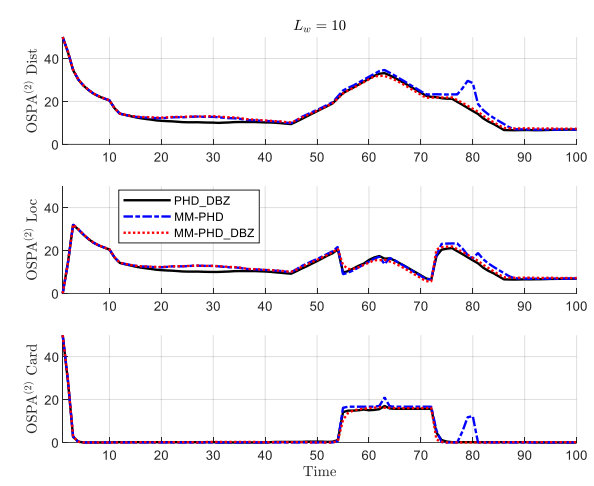

(c) $\operatorname{OSPA}^{(2)}\left(L_{w}=10\right)$
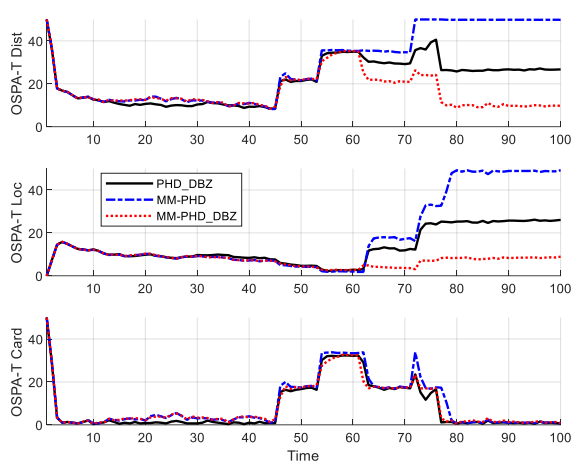

(d) OSPA-T

Fig.7. Performance comparison among different trackers (MDV=3m/s).

Fig. 8 shows OSPA-Ts of different trackers when $M D V=5 \mathrm{~m} / \mathrm{s}$. The performance curve of the MM-PHD tracker can be used as a reference. When the MDV becomes larger, the performances of the PHD_DBZ and MM-PHD_DBZ trackers are deteriorated, which is consistent with intuition, since when the number of missed detections is too large, almost all trackers are difficult to solve this problem.
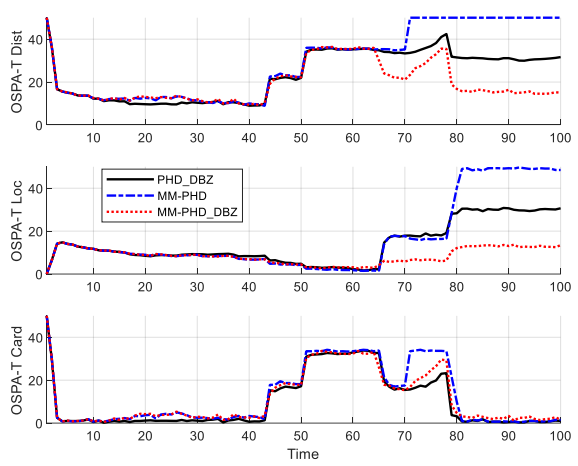

Fig.8. OSPA-Ts of different trackers (MDV=5m/s, 100 runs).

\section{CONCLUSIONS}

To track multiple maneuvering targets in the presence of the DBZ, we proposed a practical and fully functional tracker, i.e., the MM-PHD_DBZ tracker. This tracker is based on the MMPHD filter for tracking multiple maneuvering targets, and utilizes the realistic GMTI measurement model including the polar and Doppler measurements, especially the MDV incorporated detection probability model to suppress the DBZ masking. Besides, the tracker is equipped with the ATI and TLP functions, which are required for a practical tracker and also are 
needed to evaluate the overall GMTI tracking performance. Simulation results show that the proposed tracker outperforms significantly the existing works. Moreover, we pointed out that the OSPA-T, not the latest OSPA ${ }^{(2)}$, is suitable for evaluating the overall GMTI performance. It should be pointed out that, when the number of missed detections is too large, there is no tracker to solve the DBZ masking problem. Hence, some of the future works will focus on sensor control and multisensor fusion techniques since the radial velocity of a target with respect to a sensor is affected by the relative geometry between the target and the sensor.

\section{APPENDIX}

\section{A. Cart2Polar}

Function "Cart2Polar" in (5) is defined as

$\frac{[r, a, e]=\operatorname{Cart} 2 \operatorname{Polar}\left(\boldsymbol{\xi}, \boldsymbol{\xi}_{s}\right)}{\left\{\begin{array}{l}r=\left[\left(x-x_{s}\right)^{2}+\left(y-y_{s}\right)^{2}+\left(z-z_{s}\right)^{2}\right]^{1 / 2} \\ a=\operatorname{atan} 2\left[\left(y-y_{s}\right),\left(x-x_{s}\right)\right] \\ e=\arctan \left\{\left(z-z_{s}\right) /\left[\left(x-x_{s}\right)^{2}+\left(y-y_{s}\right)^{2}\right]^{1 / 2}\right\}\end{array}\right.}$

where "atan2" refers to the four-quadrant inverse tangent function.

\section{B. Polar2Cart}

For a polar measurement $\boldsymbol{y}_{p}=\left[r_{m}, a_{m}, e_{m}\right]^{\mathrm{T}}$, its corresponding Cartesian measurement $\boldsymbol{y}_{c}=\left[x_{m}, y_{m}, z_{m}\right]^{\mathrm{T}}$ can be computed via the following nonlinear transformation

$\frac{\boldsymbol{y}_{c}=\operatorname{Polar} 2 \operatorname{Cart}\left(\boldsymbol{y}_{p}, \boldsymbol{\xi}_{s}\right)}{\left\{\begin{array}{l}x_{m}=r_{m} \cos a_{m} \cos e_{m}+x_{s} \\ y_{m}=r_{m} \sin a_{m} \cos e_{m}+y_{s} \\ z_{m}=r_{m} \sin e_{m}+z_{s}\end{array}\right.}$

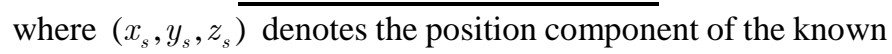
sensor state $\boldsymbol{\xi}_{s}=\left[\begin{array}{llllll}x_{s} & y_{s} & z_{s} & \dot{x}_{s} & \dot{y}_{s} & \dot{z}_{s}\end{array}\right]^{\mathrm{T}}$.

\section{Cov_Polar2Cart}

In Section IV.B, a polar measurement $\boldsymbol{y}_{p}=\left[r_{m}, a_{m}, e_{m}\right]^{\mathrm{T}}$ is transformed to the Cartesian coordinate measurement $\boldsymbol{y}_{c}$ using a classical conversion, although there exist other transformation methods, e.g., debiased transformation [65, 66], unbiased transformation $[67,68,69]$, etc. After the polar-to-Cartesian transformation, the corresponding covariance of converted measurement $\boldsymbol{y}_{c}$ is given by [70]

$$
\boldsymbol{R}_{c}\left(\boldsymbol{y}_{p}\right)=\left[\begin{array}{ccc}
\sigma_{x}^{2} & \sigma_{x y}^{2} & \sigma_{x z}^{2} \\
\sigma_{x y}^{2} & \sigma_{y}^{2} & \sigma_{y z}^{2} \\
\sigma_{x z}^{2} & \sigma_{y z}^{2} & \sigma_{z}^{2}
\end{array}\right]=\boldsymbol{J}_{p} \boldsymbol{R}_{p} \boldsymbol{J}_{p}^{\mathrm{T}}
$$

[1] W. Koch

On exploiting 'negative' sensor evidence for target tracking and sensor data fusion

Inform. Fusion, vol. 8, no. 1, pp. 28-39, 2007.

[2] W. Koch and R. Klemm

Ground target tracking with STAP radar

IEE Proc.-Radar, Sonar Navig., vol. 148, no. 3, pp. 173-185, 2001.

[3] M. Ulmke, O. Erdinc, and P. Willett

GMTI tracking via the Gaussian mixture cardinalized probability hypothesis density filter

IEEE Trans. Aerosp. Electron. Syst., vol. 46, no. 4, pp. 1821-1833, 2010. where $\boldsymbol{R}_{p}=\operatorname{blkdiag}\left(\left[\sigma_{r}^{2}, \sigma_{a}^{2}, \sigma_{e}^{2}\right]\right)$ and

$$
\boldsymbol{J}_{p}=\left[\begin{array}{ccc}
\cos a_{m} \cos e_{m} & -r_{m} \sin a_{m} \cos e_{m} & -r_{m} \cos a_{m} \sin e_{m} \\
\sin a_{m} \cos e_{m} & r_{m} \cos a_{m} \cos e_{m} & -r_{m} \sin a_{m} \sin e_{m} \\
\sin e_{m} & 0 & r_{m} \cos e_{m}
\end{array}\right]
$$

Hence, the elements of the corresponding $\boldsymbol{R}_{c}\left(\boldsymbol{y}_{p}\right)$ are given below

$$
\begin{aligned}
\sigma_{x}^{2}= & \sigma_{r}^{2} \cos ^{2} a_{m} \cos ^{2} e_{m}+\sigma_{a}^{2} r_{m}^{2} \sin ^{2} a_{m} \cos ^{2} e_{m} \\
& +\sigma_{e}^{2} r_{m}^{2} \cos ^{2} a_{m} \sin ^{2} e_{m} \\
\sigma_{x y}^{2}= & \sigma_{r}^{2} \sin a_{m} \cos a_{m} \cos ^{2} e_{m}-\sigma_{a}^{2} r_{m}^{2} \sin a_{m} \cos a_{m} \cos ^{2} e_{m} \\
& +\sigma_{e}^{2} r_{m}^{2} \sin a_{m} \cos a_{m} \sin ^{2} e_{m} \\
\sigma_{y}^{2}= & \sigma_{r}^{2} \sin ^{2} a_{m} \cos ^{2} e_{m}+\sigma_{a}^{2} r_{m}^{2} \cos ^{2} a_{m} \cos ^{2} e_{m} \\
& +\sigma_{e}^{2} r_{m}^{2} \sin ^{2} a_{m} \sin ^{2} e_{m} \\
\sigma_{x z}^{2}= & \sigma_{r}^{2} \cos a_{m} \sin e_{m} \cos e_{m}-\sigma_{e}^{2} r_{m}^{2} \cos a_{m} \sin e_{m} \cos e_{m} \\
\sigma_{y z}^{2}= & \sigma_{r}^{2} \sin a_{m} \sin e_{m} \cos e_{m}-\sigma_{e}^{2} r_{m}^{2} \sin a_{m} \sin e_{m} \cos e_{m} \\
\sigma_{z}^{2}= & \sigma_{r}^{2} \sin ^{2} e_{m}+\sigma_{e}^{2} r_{m}^{2} \cos ^{2} e_{m}
\end{aligned}
$$

\section{Jacobian matrix $\boldsymbol{H}_{f}(\cdot)$}

In (24), the fictitious measurement matrix $\boldsymbol{H}_{f}\left(\hat{\boldsymbol{\xi}}_{+}\right)$is the Jacobian matrix of $n_{c}$ with respect to $\xi$ evaluated at $\hat{\boldsymbol{\xi}}_{+}=\left[\begin{array}{llllll}\hat{x}_{+} & \hat{y}_{+} & \hat{z}_{+} & \hat{\dot{x}}_{+} & \hat{\dot{y}}_{+} & \hat{\dot{z}}_{+}\end{array}\right]^{\mathrm{T}}$, i.e.,

$$
\boldsymbol{H}_{f}\left(\hat{\boldsymbol{\xi}}_{+}\right)=-\left.\frac{\partial n_{c}}{\partial \boldsymbol{\xi}}\right|_{\xi=\hat{\boldsymbol{\xi}}_{+}}=\left[\begin{array}{llllll}
n_{1} & n_{2} & n_{3} & n_{4} & n_{5} & n_{6}
\end{array}\right]^{\mathrm{T}}
$$

where

$n_{1}=-\left(\hat{\dot{x}}_{+}-\hat{n}_{c} \cos \hat{a} \cos \hat{e}\right) / \hat{r} \quad, \quad n_{2}=-\left(\hat{\dot{y}}_{+}-\hat{n}_{c} \sin \hat{a} \cos \hat{e}\right) / \hat{r}$, $n_{3}=-\left(\hat{\dot{z}}_{+}-\hat{n}_{c} \sin \hat{e}\right) / \hat{r}, n_{4}=-\cos \hat{a} \cos \hat{e}, n_{5}=-\sin \hat{a} \cos \hat{e}$, $n_{6}=-\sin \hat{e},[\hat{r}, \hat{a}, \hat{e}]=\operatorname{Cart} 2 \operatorname{Polar}\left(\hat{\boldsymbol{\xi}}_{+}, \boldsymbol{\xi}_{s}\right), \hat{n}_{c}=n_{c}($

\section{E. Product formula for Gaussian densities}

Given $\boldsymbol{H}, \boldsymbol{R}, \boldsymbol{m}$, and $\boldsymbol{P}$ of appropriate dimensions and that $\boldsymbol{R}$ and $\boldsymbol{P}$ are positive definite, then [4][27]

$$
\mathcal{N}(\boldsymbol{z} ; \boldsymbol{H} \boldsymbol{x}, \boldsymbol{R}) \mathcal{N}(\boldsymbol{x} ; \boldsymbol{m}, \boldsymbol{P})=\mathcal{N}(\boldsymbol{z} ; \boldsymbol{H} \boldsymbol{m}, \boldsymbol{S}) \mathcal{N}(\boldsymbol{x} ; \tilde{\boldsymbol{m}}, \tilde{\boldsymbol{P}})
$$

with

$$
\boldsymbol{S}=\boldsymbol{H} \boldsymbol{P} \boldsymbol{H}^{\mathrm{T}}+\boldsymbol{R} \text {, and } \boldsymbol{G}=\boldsymbol{P} \boldsymbol{H}^{\mathrm{T}} \boldsymbol{S}^{-1} \text {. }
$$$$
\tilde{\boldsymbol{P}}=\boldsymbol{P}-\boldsymbol{G} \boldsymbol{S} \boldsymbol{G}^{\mathrm{T}}
$$

\section{REFERENCES}

[4] W. H. Wu, W. J. Liu, J. Jiang, L. Gao, Q. Wei, and C. Liu GM-PHD filter-based multi-target tracking in the presence of Doppler blind zone

Digit Signal Process., vol. 52, pp. 1-12, 2016.

[5] Y.Ruan and P.Willett The turbo PMHT

IEEE Trans. Aerosp. Electron. Syst., vol. 40, no. 4, pp. 1388-1398, 2004.

[6] W. Koch

GMTI-tracking and information fusion for ground surveillance In Proc. SPIE Signal Data Process. Small Targets, 2001, pp. 381-392.

[7] M. Mertens and R. Kohlleppel 
Ground target tracking with experimental data of the PAMIR system In Proc. 17th Int. Conf. Inf. Fusion, 2014, pp. 1-8.

[8] M. Mertens, W. Koch, and T. Kirubarajan Exploiting Doppler blind zone information for ground moving target tracking with bistatic airborne radar

IEEE Trans. Aerosp. Electron. Syst., vol. 50, no. 1, pp. 130-148, 2014.

[9] M. Mertens and U. Nickel

GMTI tracking in the presence of Doppler and range ambiguities In Proc. 14th Int. Conf. Inf. Fusion, 2011, pp. 1-8.

[10] M. Ulmke and W. Koch Road-map assisted ground moving target tracking IEEE Trans. Aerosp. Electron. Syst., vol. 42, no. 4, pp. 1264-1274, 2006.

[11] N. Gordon and B. Ristic

Tracking airborne targets occasionally hidden in the blind Doppler Digit. Signal Process., vol. 12, no. 12, pp. 383-393, 2002.

[12] J. Clark, P. Kountouriotis, and R. Vinter A new Gaussian mixture algorithm for GMTI tracking under a minimum detectable velocity constraint IEEE Trans. Autom. Control, vol. 54, no. 12, pp. 2745-2756, 2009.

[13] D. A. Zaugg, A. A. Samuel, D. E. Waagen, and H. A. Schmitt A combined particle/Kalman filter for improved tracking of beam aspect targets In IEEE Workshop on Statist. Signal Process., 2003, pp. 535-538.

[14] M. Yu, C. Liu, B. Li, and W.-H. Chen An enhanced particle filtering method for GMTI radar tracking IEEE Trans. Aerosp. Electron. Syst., vol. 52, no. 3, pp. 1408-1420, 2016.

[15] L. Gong and M. Yu

A new Gaussian mixture method with exactly exploiting the negative information for GMTI radar tracking in a low-observable environment Aerosp. Sci. Technol., vol. 80, pp. 1-10, 2018.

[16] H. Huang, R. Yang, P. Foo, G. Ng, M. Mertens, M, Ulmke, and W. Koch Convoy tracking in Doppler blind zone regions using GMTI radar In Proc. 16th Int. Conf. Inf. Fusion, 2013, pp. 1768-1775.

[17] T. Kirubarajan and Y. Bar-Shalom

Tracking evasive move-stop-move targets with a GMTI radar using a VSIMM estimator

IEEE Trans. Aerosp. Electron. Syst., vol. 39, no. 3, pp. 1098-1103, 2003.

[18] S. Zhang and Y. Bar-Shalom

Tracking move-stop-move targets with state-dependent mode transition probabilities

IEEE Trans. Aerosp. Electron. Syst., vol. 47, no. 3, pp. 2037-2054, 2011.

[19] M. Hernandez, A. Benavoli, A. Graziano, A. Farina, and M. Morelande Performance measures and MHT for tracking move-stop-move targets with MTI sensors

IEEE Trans. Aerosp. Electron. Syst., vol. 47, no. 2, pp. 996-1025, 2011.

[20] T. Song, D. Musicki, and Y. Kim

Tracking through occlusions and track segmentation reduction

IEEE Trans. Aerosp. Electron. Syst., vol. 49, no. 1, pp. 623-631, 2013.

[21] L. Lin, Y. Bar-Shalom, and T. Kirubarajan

New assignment-based data association for tracking move-stop-move targets

IEEE Trans. Aerosp. Electron. Syst., vol. 40, no. 2, pp. 714-725, 2004.

[22] S. W. Yeom, T. Kirubarajan, and Y. Bar-Shalom

Track segment association, fine-step IMM and initialization with Doppler for improved track performance

IEEE Trans. Aerosp. Electron. Syst., vol. 40, no. 1, pp. 293-309, 2004.

[23] S. Zhang and Y. Bar-Shalom

Track segment association for GMTI tracks of evasive move-stop-move maneuvering targets

IEEE Trans. Aerosp. Electron. Syst., vol. 47, no. 3, pp. 1899-1914, 2011.

[24] X. Li and V. Jilkov

Survey of maneuvering target tracking. Part V: Multiple-model methods IEEE Trans. Aerosp. Electron. Syst., vol. 41, no. 4, pp. 1255-1321, 2005.

[25] D. B. Reid

An algorithm for tracking multiple targets

IEEE Trans. Autom. Control, vol. 24, no. 6, pp. 843-854.

[26] T. E. Fortmann, Y. Bar-shalom, M. Scheffe Multi-target tracking using joint probabilistic data association In 19th IEEE Conf. on Decision and Control, 1980, pp. 807-812.

[27] B. N. Vo and W. K. Ma

The Gaussian mixture probability hypothesis density filter IEEE Trans. Signal Process., vol. 54, no. 11, pp. 4091-4104, 2006.

[28] B. T. Vo, B. N. Vo, and A. Cantoni Analytic implementations of the cardinalized probability hypothesis density filter
IEEE Trans. Signal Process., vol. 55, no. 7, pp. 3553-3567, 2007.

[29] B. T. Vo, B. N. Vo, and A. Cantoni

The cardinality balanced multi-target multi-Bernoulli filter and its implementations

IEEE Trans. Signal Process., vol. 57, no. 2, pp. 409-423, 2009.

[30] S. Reuter, B. T. Vo, B. N. Vo, and K. Dietmayer

The labeled multi-Bernoulli filter

IEEE Trans. Signal Process., vol. 62, no. 12, pp. 3246-3260, 2014.

[31] F. Papi, B.-N. Vo, B.-T. Vo, C. Fantacci, and M. Beard Generalized labeled multi-Bernoulli approximation of multi-object densities

IEEE Trans. Signal Process., vol. 63, no. 20, pp. 5487-5497, 2015.

[32] B. T. Vo and B. N. Vo

Labeled random finite sets and multi-object conjugate priors IEEE Trans. Signal Process., vol. 61, no. 13, pp. 3460-3475, 2013.

[33] B.-N. Vo, B.-T. Vo, and D. Phung Labeled random finite sets and the Bayes multi-target tracking filter IEEE Trans. Signal Process., vol. 62, no. 24, pp. 6554-6567, 2014.

\section{[34] R. Mahler}

Advances in statistical multisource-multitarget information fusion Norwood, MA, USA: Artech House, 2014.

[35] B.-N. Vo, B.-T. Vo, and H.-G. Hoang

An efficient implementation of the generalized labeled multi-Bernoulli Filter

IEEE Trans. Signal Process., vol. 65, no. 8, pp. 1975-1987, 2017.

[36] K. Punithakumar, T. Kirubarajan, and A. Sinha Multiple-model probability hypothesis density filter for tracking maneuvering targets

IEEE Trans. Aerosp. Electron. Syst., vol. 44, no. 1, pp. 87-98, 2008.

[37] S. Pasha, B.-N. Vo, H. D. Tuan, and W. K. Ma A Gaussian mixture PHD filter for jump Markov system models IEEE Trans. Aerosp. Electron. Syst., vol. 45, no. 3, pp. 919-936, 2009.

[38] T. M. Wood Interacting methods for manoeuvre handling in the GM-PHD filter IEEE Trans. Aerosp. Electron. Syst., vol. 47, no. 4, pp. 3021-302, 2011.

[39] R. Sithiravel, M. Mcdonald, B. Balaji, and T. Kirubarajan Multiple model spline probability hypothesis density filter IEEE Trans. Aerosp. Electron. Syst., vol. 52, no. 3, pp. 1210-1226, 2016.

[40] R. Georgescu and P. Willett The multiple model CPHD tracker

IEEE Trans. Signal Process., vol. 60, no. 4, pp. 1741-1751, 2012.

[41] R. Mahler

On multitarget jump-markov filters

In 15th Int. Conf. Inf. Fusion, 2012, pp. 149-156.

[42] D. Dunne and T. Kirubarajan

Multiple model multi-Bernoulli filters for manoeuvering targets

IEEE Trans. Aerosp. Electron. Syst., vol. 49, no. 4, pp. 2679-2692, 2013.

[43] J. Yang, H. Ji, and H. Ge

Multi-model particle cardinality-balanced multi-target multi-Bernoulli algorithm for multiple manoeuvring target tracking

IET Radar, Sonar Navig., vol. 7, no. 2, pp. 101-112, 2013.

[44] S. Reuter, A. Scheel, and K. Dietmayer

The multiple model labeled multi-Bernoulli filter In 18th Int. Conf. Inf. Fusion, 2015, pp. 1574-1580.

[45] Y. Punchihewa, B.-N. Vo, and B.-T. Vo A generalized labeled multi-Bernoulli filter for maneuvering targets In 19th Int. Conf. Inf. Fusion, 2016.

[46] Y. Punchihewa

Efficient generalized labeled multi-Bernoulli filter for jump markov system

In Int. Conf. Contr., Automa. Inf. Sci. (ICCAIS), 2017.

[47] W. Yi, M. Jiang, and R. Hoseinnezhad The multiple model Vo-Vo filter

IEEE Trans. Aerosp. Electron. Syst., vol. 53, no. 2, pp. 1045-1054, 2017.

[48] M. Mertens, M. Ulmke, and W. Koch

Ground Target Tracking With RCS Estimation Based on Signal Strength Measurements

IEEE Trans. Aerosp. Electron. Syst., vol. 52, no. 1, pp. 205-220, 2016.

[49] R. Kohlleppel

Ground moving target tracking of PAMIR detections with a Gaussian mixture-PHD filter

In Proc. Int. Radar Symp., 2011, pp. 193-198.

[50] M. Yu, H. Oh, and W. Chen

An improved multiple model particle filtering approach for manoeuvring target tracking using airborne GMTI with geographic information 
Aerosp. Sci. Technol., vol. 52, pp. 62-69, 2016.

[51] S. Du, Z. Shi, W. Zang, et al.

Using interacting multiple model particle filter to track airborne targets hidden in blind Doppler

Journal of Zhejiang University Science A, vol. 8, no. 8, pp. 1277-1282, 2007.

[52] J. Clark, P. Kountouriotis, and R. Vinter

A Gaussian mixture GMTI tracker: extensions for multiple target models and clutter

Imperial College London, London, U.K., C\&P Tech. Rep. 08/RBV1, 2008.

[53] Y. Bar-Shalom, F. Daum, and J. Huang

The probabilistic data association filter

IEEE Control Systems Magazine, vol. 29, no. 6, pp. 82-100, 2009.

[54] M. Beard, B.-T. Vo, B.-N. Vo

Performance evaluation for large-scale multi-target tracking algorithms In Int. Conf. Inf. Fusion, 2018.

[55] B. Ristic, B.-N. Vo, D. Clark, and B.-T. Vo A metric for performance evaluation of multi-target tracking algorithms IEEE Trans. Signal Process., vol. 59, no. 7, pp. 3452-3457, 2011.

[56] X. Xie, H. Sun, W. Wu, S. Jiang, and J. Yuan

MM-GM-PHD Filter-Based Maneuvering Target Tracking in the presence of Doppler Blind Zone

In IEEE 3rd Information Technology, Networking, Electronic and Automation Control Conference (ITNEC), 2019.

[57] W. H. Wu, H. Sun, Y. Cai, S. Jiang, and J. Xiong, "Tracking multiple maneuvering targets hidden in the DBZ based on the MM-GLMB filter," IEEE Trans. Signal Process., vol. 68, pp. 2912-2924, 2020, DOI: 10.1109/TSP.2020.2988635.

[58] M. Mallick and S. Arulampalam Comparison of Nonlinear Filtering Algorithms in Ground Moving Target Indicator (GMTI) Target Tracking In Proc. SPIE, 2003

[59] W. H. Wu, J. Jiang, W. Liu, X. Feng, L. Gao, and X. Qin Augmented state GM-PHD filter with registration errors for multi-target tracking by Doppler radars

Signal Process., vol. 120, pp. 117-128, 2016.

[60] X. Wang, D. Musicki, R. Ellem, and F. Fletcher
Efficient and enhanced multi-target tracking with Doppler measurements IEEE Trans. Aerosp. Electron. Syst., vol. 45, no. 4, pp. 1400-1417, 2009.

[61] K. Y. Li, U. Pillai, and S. Mudaliar

Minimum detectable velocity (MDV) for ATI and STAP for high speed platforms

In Proc. IEEE Int. Radar Conf., 2019.

[62] J. Yoon, D. Kim, S. Bae, et al.

Joint initialization and tracking of multiple moving objects using Doppler information

IEEE Trans. Signal Process., vol. 59, no. 7, pp. 3447-3452, 2011.

[63] K. Panta, D. E. Clark, B.-N. Vo

Data association and track management for the Gaussian mixture probability hypothesis density filter

IEEE Trans. Aerosp. Electron. Syst., vol. 45, no. 3, pp. 1003-1016, 2009.

[64] D. Schuhmacher, B. T. Vo, and B. N. Vo

A consistent metric for performance evaluation of multi-object filters IEEE Trans. Signal Process., vol. 56, no. 8, pp. 3447-3457, 2008.

[65] D. Lerro and Y. Bar-Shalom

Tracking with debiased consistent converted measurements versus EKF

IEEE Trans. Aerosp. Electron. Syst., vol. 29, no. 3, pp. 1015-1022, 1993.

[66] S. Piotr

Explicit expressions for debiased statistics of 3D converted measurements

IEEE Trans. Aerosp. Electron. Syst., vol. 35, no. 1, pp. 368-370, 1999.

[67] L. Mo, X. Song, Y. Zhou, Z. Sun, and Y. Bar-Shalom

Unbiased converted measurements for tracking

IEEE Trans. Aerosp. Electron. Syst., vol. 34, no. 3, pp. 1023-1027, 1998.

[68] Z. Duan, C. Han, and X. R. Li Comments on "Unbiased Converted Measurements for Tracking" IEEE Trans. Aerosp. Electron. Syst., vol. 40, no. 4, pp. 1374-1377, 2004.

[69] S. V. Bordonaro, P. Willett, and Y. Bar-Shalom Unbiased tracking with converted measurements In Proc. IEEE Int. Radar Conf., 2012, pp. 0741-0745.

[70] W. H. Wu, J. Jiang, W. Liu, X. Qin, and W. Zhang A sequential converted measurement Kalman filter in the ECEF coordinate system for airborne Doppler radar Aerosp. Sci. Technol., vol. 51, pp. 11-17, 2016. 